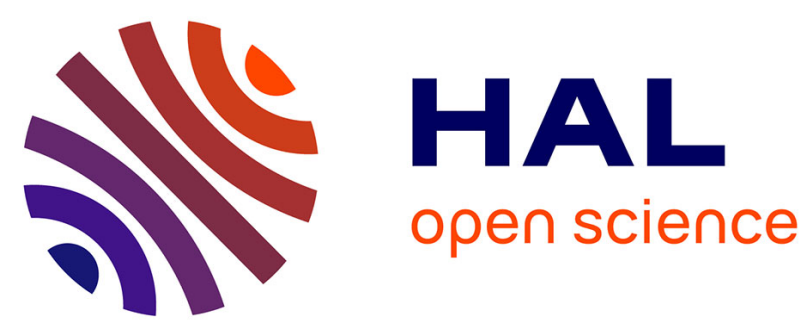

\title{
What can we learn from observed temperature and salinity isopycnal anomalies at eddy generation sites? Application in the Tropical Atlantic Ocean
}

Micael Aguedjou, A Chaigneau, I Dadou, Yves Morel, C Pegliasco, C y Da-Allada, E Baloïtcha

\section{To cite this version:}

Micael Aguedjou, A Chaigneau, I Dadou, Yves Morel, C Pegliasco, et al.. What can we learn from observed temperature and salinity isopycnal anomalies at eddy generation sites? Application in the Tropical Atlantic Ocean. Journal of Geophysical Research. Oceans, 2021, 10.1029/2021JC017630 . hal-03368392

\section{HAL Id: hal-03368392 \\ https://hal.science/hal-03368392}

Submitted on 6 Oct 2021

HAL is a multi-disciplinary open access archive for the deposit and dissemination of scientific research documents, whether they are published or not. The documents may come from teaching and research institutions in France or abroad, or from public or private research centers.
L'archive ouverte pluridisciplinaire HAL, est destinée au dépôt et à la diffusion de documents scientifiques de niveau recherche, publiés ou non, émanant des établissements d'enseignement et de recherche français ou étrangers, des laboratoires publics ou privés. 
1 What can we learn from observed temperature and salinity isopycnal anomalies at eddy generation sites? Application in the Tropical Atlantic Ocean

4 Aguedjou H. M. A. ${ }^{1,2, *}$, A. Chaigneau ${ }^{1,3,2}$, I. Dadou ${ }^{1}$, Y. Morel $^{1}$, C. Pegliasco ${ }^{4}$, C. Y. Da-Allada ${ }^{2,3,5}$, E. Baloïtcha ${ }^{2}$ 5

${ }^{1}$ LEGOS, University of Toulouse, CNRS, IRD, CNES, UT3, Toulouse, France

$8 \quad{ }^{2}$ CIPMA/UAC, Cotonou, Benin

$9 \quad{ }^{3}$ IRHOB, Cotonou, Benin

$10 \quad{ }^{4}$ CLS, Ramonville Saint-Agne

$11 \quad{ }^{5}$ UNSTIM, Abomey, Benin

12 *micael.aguedjou@legos.obs-mip.fr aguhabib2000@gmail.com

13

14

15 Key points:

16

17

18

- At their generation site, $50 \%$ of observed eddies have non-significant isopycnal temperature/salinity $(\theta / S)$ anomalies in the TAO.

- On density-coordinates, both CEs and AEs can exhibit significant positive, negative or non-significant isopycnal $\theta / S$ anomalies.

- We discuss the relationship between isopycnal $\theta / S$ and PV anomalies and how they can inform us on their generation mechanisms. 


\section{Abstract:}

Based on 18 years of satellite altimetry data and temperature/salinity $(\theta / S)$ profiles from Argo floats, we analyze the isopycnal $\theta / S$ anomalies $\left(\theta^{\prime} / S^{\prime}\right)$ within new-born eddies in the tropical Atlantic Ocean (TAO). Our results show that on density-coordinates, both anticyclonic eddies (AEs) and cyclonic eddies (CEs) can exhibit

31 positive, negative or non-significant $\theta^{\prime} / S^{\prime}$. Almost half of the sampled eddies do not have significant $\theta^{\prime} / S^{\prime}$ at their 32 generation site. The other half exhibits significant positive or negative $\theta^{\prime}$, up to $\pm 0.5{ }^{\circ} \mathrm{C}$ typically. More than $3370 \%$ of them have a subsurface signature. Refined analyses of the vertical structure of new-born eddies in three 34 selected subregions show the dominance of cold (warm) subsurface AEs (CEs) likely due to isopycnal advection 35 of large scale potential vorticity (PV) and $\theta$. PV is a key parameter to analyze eddy generation and dynamics. 36 Isopycnal advection, friction or diapycnal mixing can be involved in the generation of PV anomalies from which 37 vortices can then emerge. However, it is difficult to evaluate PV at mesoscale. In this study we propose to 38 combine $\theta^{\prime} / S^{\prime}$ and PV anomalies to analyze which process could be involved in its transformation in the ocean.

39 We argue that eddies created by diapycnal mixing or isopycnal advection of water-masses are associated with $40 \mathrm{PV}$ anomalies and significant $\theta^{\prime} / S^{\prime}$. In contrast, a frictional forcing also creates PV anomalies but without 41 modifying $\theta / S$. Even though our results remain qualitative, the proposed diagnostics can be of interest to validate 42 realistic models and then use them to analyze the PV anomaly sources.

44 Keywords: mesoscale eddies; isopycnal temperature/salinity anomalies; eddy generation mechanisms; potential vorticity; tropical Atlantic Ocean. 


\section{Plain Language Summary}

Mesoscale vortices are common features in the global ocean, having typical length scales of 10 to 100

55

56

57 8 9 0 1 2 3 4 5 6 7 $\mathrm{km}$ and lifespans from several weeks to several months or even years for the more energetic structures. The vorticity of these quasi-circular rotating structures emerging from large-scale currents can be formed by several physical processes: isopycnal advection of a water mass, friction (wind stress, bottom stress) or diapycnal mixing. In this study, we first analyze the vertical structure of temperature/salinity in the eddies at their generation site. Based on the analysis of satellite and in-situ data in the tropical Atlantic Ocean, we show that $\sim 50 \%$ of the eddies do not present significant isopycnal temperature anomalies when they are generated. In contrast, the remaining $\sim 50 \%$ of eddies present significant isopycnal temperature anomalies, but with unexpected numerous cold anticyclones and warm cyclones. We then discuss the link between the potential vorticity and isopycnal temperature anomalies, showing combinations of the previous physical processes involved have to be invoked to explain the observed eddy characteristics. (1) 66 67

\section{Introduction}


The Tropical Atlantic Ocean (TAO) is a key region for the inter-hemispheric exchange of heat, salt and

80

81

82

83

84

mass by thermohaline circulation, large-scale currents and mesoscale eddies (e.g. Thomas and Zhai, 2013; Saenko et al., 2018). In the TAO, the upper ocean circulation is mainly composed of i) equatorial limbs of the North and South Atlantic anticyclonic subtropical gyres, ii) zonal equatorial currents and iii) near-coastal current systems (Fig. 1). In the surface layer of the North and South Atlantic subtropical gyres, the salty North and South Atlantic Waters (NAW and SAW, respectively; Fig. 1a) have maximum salinities exceeding 37 in their formation region with densities of $\sim 25.0$ - $25.5 \mathrm{~kg} \mathrm{~m}^{-3}$ (Tsuchiya et al., 1992; Bourlès et al., 1999a; Stramma and Schott, 1999; Stramma et al., 2005a-b Kirchner et al. 2009). In contrast, in the surface layer of the equatorial Atlantic, is found the relatively warm and fresh Tropical Surface Water (TSW; Fig. 1a) with densities lower than $24.5 \mathrm{~kg} \mathrm{~m}^{-3}$ (e.g. Tsuchiya et al., 1992, Tomczak and Godfrey, 1994 Stramma and Schott, 1999 ; Stramma et al., 2005a-b). Fraction of NAW and SAW is advected west-equatorward by the North Equatorial Current (NEC) and the southern South Equatorial Current (sSEC), respectively (Fig. 1a). In the equatorial region this fraction subducts and spreads below the lighter TSW forming a subsurface S maximum in the upper thermocline, known as Subtropical Underwater (STUW; Fig 1b) within the density range $\sigma_{\theta} \sim 24.5-26.3 \mathrm{~kg} \mathrm{~m}^{-3}$ (Snowden and Molinari, 2003; Tsuchiya et al., 1992; Stramma and Schott, 1999; Stramma et al., 2005b). In the upper thermocline of the eastern TAO, another salinity maximum water, known as East Atlantic Water (EAW), is observed, but noticeably fresher than the STUW (Wilson et al., 1994; Bourlès et al., 1999a; Urbano et al. 2008; Kirchner et al. 2009).

Underneath the surface waters are found the North and South Atlantic Central Waters (NACW and SACW, respectively; Fig 1b). They are characterized by a linear temperature-salinity $(\theta-S)$ relationship in the density range $\sigma_{\theta} \sim 25.8$ - $27.1 \mathrm{~kg} \mathrm{~m}^{-3}$ (e.g. Emery, 2003; Liu and Tanhua, 2019) and are connected at around 15 N (Sverdrup et al. 1942; Emery and Meincke, 1986; Stramma and Schott, 1999; Stramma et al., 2005b). Below the base of the pycnocline $\left(\sigma_{\theta}>26.0 \mathrm{~kg} \mathrm{~m}^{-3}\right)$, water-masses present $\theta$-S properties close to the Atlantic Subarctic Intermediate Water (ASIW) in the Northern TAO and Antarctic Intermediate Water (AAIW) in the Southern TAO (e.g. Emery, 2003). 
Although the relatively complex large-scale circulation shown in Fig. 1 is important for the

105

106

107

108

109

110

111

112

113

114

115

116

117

118

119

120

121

122

123

124

125

126

127

128

129

redistribution of water-masses, mesoscale eddies are also known to play a key role in the transfer and redistribution of energy, heat, salt and physical/biogeochemical properties from their generation regions to their dissipation sites (e.g. Chaigneau et al., 2011; Gaube et al., 2014; McGillicuddy 2016). They contribute to the mixing and redistribution of water-masses through several mechanisms such as eddy horizontal stirring, eddyinduced upwelling/downwelling, subduction or trapping and self-advection over long distances across the basin (e.g. McWilliams and Flierl, 1979; Herbette et al, 2004; Chelton et al., 2011), being able to connect eastern and western boundaries (e.g., Laxenaire et al., 2018).

Eddy evolutions also strongly depend on their dynamical properties, in particular their potential vorticity (PV) signature which is a key quantity to analyze the formation and dynamics of eddies. Indeed, the core of an eddy in geostrophic equilibrium is necessarily associated with a significant isopycnal PV anomaly, from which the vorticity or stratification of the eddy core can be determined (Hoskins, 1985; Morel and McWilliams 1997; Herbette et al., 2003). Since PV is also conserved for fluid particles in adiabatic and frictionless evolution, the formation of a PV anomaly (and of an eddy subsequently) is necessarily associated with either adiabatic displacement of particles in a background PV gradient, or friction or diapycnal mixing (including the diabatic transformation associated with atmospheric heat and salt fluxes). Many studies have shown how vortices can be formed by the displacement of particles in a background PV gradient. Meridional advection on the planetary beta-plane (Wang, 2005), interaction of currents with sea-mounts, islands, or continental shelves (Aristégui et al., 1994; Herbette et al, 2004) can also be interpreted as creation of PV anomalies by isopycnal advection of particles. These processes are thought to play a significant role in the formation of mesoscale eddies at least in some regions of the TAO, such as east of the North Brazil Current (NBC) retroflection (e.g. Aguedjou et al., 2019). Some recent studies have shown that non-conservative forcings could also lead to the generation of strong PV anomalies and vortices. Indeed, theoretical and numerical studies have shown that diapycnal mixing (Haynes and McIntyre, 1987; 1990; Morel and McWilliams, 2001) and frictional effects, associated with lateral viscous layers (D’Asaro, 1988; Morel and McWilliams 2001; Akuetevi and Wirth, 2015), the wind (Thomas, 2005; Morel et al, 2006; Holmes et al, 2014; Holmes and Thomas, 2016) or with the bottom boundary layer 
130 (Benthuysen and Thomas, 2012; Gula et al, 2015; 2016; Morvan et al, 2019), are all efficient mechanisms that

131 can modify PV, create PV anomalies and subsequently vortical structures (Morel et al, 2019; Assene et al, 2020).

132 Finally, also note that the well-known formation of eddies by barotropic and/or baroclinic instabilities of mean

133 currents is related to the existence of isopycnal PV gradient of opposite signs and can be interpreted as the result

134 of the creation of opposite sign PV anomalies or dipolar vortical structures (Charney and Stern, 1962; Morel and

135 McWilliams, 2001). The mechanisms responsible for the determination of the PV structure of the unstable mean

136 current is thus of fundamental importance too.

137 If, as noted above, there now exist several studies analyzing the origin of the processes (adiabatic,

138 frictional or diapycnal mixing) involved in the generation of PV anomalies and subsequent formation of eddies

139 in numerical simulations, it remains to be evaluated in nature. This is important because friction and diapycnal

140 mixing are parameterized in numerical models, so numerical results have to be taken cautiously as long as they

141 are not confirmed by observations. However, this is very challenging since the calculation of PV and its

142 evolution require a three-dimensional description of currents and stratification. As such, the detailed diagnostics

143 of PV evolution done in numerical simulation are impossible to reproduce for the real ocean. However, in the

144 present study we propose to separate mechanisms depending on their combined effects on the transformation of

$145 \mathrm{PV}$ and transformation of $\theta / S$. Indeed, it has been shown that diapycnal mixing or advection of water masses can

146 lead to significant PV modification accompanied by isopycnal $\theta / S$ anomalies $\left(\theta^{\prime} / S^{\prime}\right)$, whose sign and strength can

147 be evaluated from in-situ data. In contrast, the effect of frictional forcings is more difficult to evaluate but

148 friction is expected to create PV anomalies too with very weak isopycnal $\theta^{\prime} / S^{\prime}$. We thus argue that isopycnal $\theta^{\prime} / S^{\prime}$

149 in vortex cores, where we know there exists significant PV anomalies, can be used to analyze some mechanisms

150 at the origin of the transformation of PV in the real ocean, and represent a first step for the validation of

151 numerical models in this respect.

152 In this study, based on observations, we thus propose to combine in-situ $\theta / S$ measurements with satellite

153 altimetry data to estimate the isopycnal temperature/salinity anomalies $\left(\theta^{\prime} / S^{\prime}\right)$ of eddy cores at their generation

154 sites in the TAO. In Section 2 we describe the datasets and methods used to characterize $\theta^{\prime} / S^{\prime}$ inside eddies. In

155 Section 3 we first present $\theta / S$ characteristics of the large-scale water-masses over selected isopycnal levels. 
156

Second, we show case studies of $\theta^{\prime} / S^{\prime}$ inside individual eddies to illustrate that $\theta^{\prime} / S^{\prime}$ computed from isopycnal levels can strongly differ from the ones obtained along isobaric levels. Third, we characterize the $\theta / S$ isopycnal structure of eddies in the entire TAO and focus in some particular areas of the northern TAO. These diagnostics help i) to estimate the fraction of eddies that can participate to the trapping and redistribution of heat and salt through isopycnal advection in the TAO and ii) to depict the mean isopycnal $\theta^{\prime} / S^{\prime}$ in surface and subsurface newborn eddies. Finally, the possibility to infer where diapycnal mixing, isopycnal advection and/or frictional effects could play a significant role in the generation of PV anomalies forming eddy cores and determining their dynamical characteristics is discussed in Section 4, as well as the coherency of this information with the known dynamical features of the TAO.

\section{Data and methods}

\subsection{Altimetry data and eddy tracking}

Mesoscale eddies are identified and tracked in the TAO from daily maps of the Salto/Duacs absolute dynamic topography (ADT) gridded product. This multimission satellite altimetry product, was optimally interpolated onto a $0.25^{\circ} \times 0.25^{\circ}$ longitude/latitude daily grid (Ducet et al., 2000; Le Traon et al., 1998; Duacs/AVISO+, 2018; Pujol et al., 2016) and is freely distributed by the Copernicus Marine Environment Monitoring Service (http://marine.copernicus.eu/).

Eddies were identified from January 2000 to December 2017, using the widely used algorithm developed by Chaigneau et al. (2008; 2009). An eddy is identified by its center, corresponding to a local extremum in ADT, being maximum for an anticyclonic eddy (AE) and minimum for a cyclonic eddy (CE), and its external edge which corresponds to the outermost closed ADT contour around each detected eddy center. Eddy trajectories are constructed according to their polarity (cyclonic or anticyclonic) using the algorithm developed by Pegliasco et al. (2015). Briefly, this algorithm considers as part of the same trajectory, overlapping eddies with the same polarity detected at time $t$ and $t+1$ day. If several eddies overlap, a cost function is computed to determine the most similar eddy at the time $t+1$ day. When no overlapping eddy is found neither 
181 at time $t+1$ day nor $t+2$ day, the trajectory is stopped and the eddy is considered as dissipated. This eddy 182 tracking algorithm is also able to successfully identify eddy-merging or eddy-splitting events (16\% of the long 183 lived tracked eddies) that frequently occur in the ocean (e.g. Pegliasco et al., 2015; Laxenaire et al., 2018). As in 184 Aguedjou et al. (2019), in order to consider only long-lived and coherent structures, we retained eddies lasting

185 more than 30 days and having amplitudes and radii greater than $1 \mathrm{~cm}$ and $30 \mathrm{~km}$, respectively. A total of 7800 186 long-lived AEs and 8100 long-lived CEs were detected in the TAO between 2000 and 2017. Readers interested 187 in more detail regarding the main characteristics and seasonality of these eddies are invited to refer to Aguedjou 188 et al. (2019).

\subsection{Argo data}

\subsubsection{Argo data processing}

The vertical/isopycnal structure of mesoscale eddies is investigated using $\theta / S$ profiles acquired by Argo

192 floats in the TAO during the 2000-2017 period. These data were collected and made freely available by the 193 Coriolis project and programs that contribute to it (http://www.coriolis.eu.org). In the TAO, a total of 144033 $194 \quad \theta / S$ profiles, flagged as good or probably good by the Coriolis Data Center, were quality controlled following 195 Chaigneau et al. (2011) and Pegliasco et al. (2015). First, we retained profiles for which: i) the shallowest data is 196 not deeper than $15 \mathrm{~m}$ depth and the deepest acquisition is below $950 \mathrm{~m}$ depth, ii) at least 30 data are available 197 between the surface and $950 \mathrm{~m}$ depth and iii) the depth difference between two consecutive data does not exceed 198 a given threshold ( $25 \mathrm{~m}$ for the $0-150 \mathrm{~m}$ layer, $50 \mathrm{~m}$ for the $150-300 \mathrm{~m}$ layer, $75 \mathrm{~m}$ for the 300-500 $\mathrm{m}$ layer and $199100 \mathrm{~m}$ below $500 \mathrm{~m}$ depth). Second, each profile was visually inspected and was systematically discarded if it 200 presented a suspicious $\theta / S$ profile. The remaining 114440 profiles (Fig. 2a), representing $\sim 80 \%$ of the original 201 database, were then linearly interpolated every $5 \mathrm{~m}$ from the surface to $300 \mathrm{~m}$ depth, and every $10 \mathrm{~m}$ below. We here assumed that the shallowest values (within 0-15 $\mathrm{m}$ depth) were representative of the surface values. The 203 spatial distribution of the retained Argo profiles is rather homogeneous in the TAO, except along continental 204 shelves or around islands where Argo floats cannot reach their nominal parking depths (Fig. 2a). About 55\% of 
206 Argo float deployments in this region. The temporal distribution of valid Argo profiles in the TAO shows a 207 linear increase from 200 yearly profiles in 2000 to 12000 yearly profiles after 2017 (Fig. 2b). 224 floats.

\subsubsection{Argo data within new-born eddies}

The 114440 retained $\theta / S$ profiles were then classified into three categories depending on whether Argo floats surfaced within AEs or CEs (detected from altimetry) or outside eddies. However, along their trajectories, TAO eddies exhibit a three-step evolution composed of growth, mature and decay phases (Pegliasco et al., 2015; Sun et al., 2018; Aguedjou et al., 2019). During the growth phase of the eddies, which extends over the first $\sim 30 \%$ of their lifespan, their radius, amplitude, and kinetic energy strongly increase (Pegliasco et al., 2015; Sun et al., 2018; Aguedjou et al., 2019). Also during this phase, eddies traveled on average $200 \mathrm{~km}$ from their generation sites (See Appendix A3 and Fig. A4d). Thus, in order to investigate the isopycnal structure of newborn eddies, close to their generation sites, and infer the physical mechanisms involved in their generation, we only retained $\theta / S$ profiles acquired by Argo floats that surfaced within eddies located closer than $200 \mathrm{~km}$ from their formation sites (Fig. 2c). Around $~ 25 \%$ of the $~ 16000$ long-lived eddy trajectories were sampled within $200 \mathrm{~km}$ from their birth sites and are thus retained for the analysis of the thermohaline structure of new-born eddies presented in Section 3.4. The spatial distribution of the Argo floats that sampled these new-born eddies is shown in Fig. 2c. Note that varying the distance between $100 \mathrm{~km}$ and $300 \mathrm{~km}$ do not change the main results and discussion drawn in this study (not shown). We thus selected $200 \mathrm{~km}$, which is a good compromise between being close enough from the eddy formation sites and having a satisfactory number of eddies sampled by Argo

Figure 2d shows the mean distribution of Argo floats that surfaced within new-born eddies, as a function of the normalized radial distance from eddy centers. A normalized distance equals to 1 corresponds to the equivalent eddy radius inferred from the area enclosed by the ADT contour of the eddy-edge. Within these newborn eddies, 50\% (75\%, respectively) of the Argo floats surfaced within a normalized distance of 2/3 (0.9) the eddy equivalent radius from the eddy center (Fig. 2d). About 20\% surfaced at a normalized radial distance 
higher than 1 . This occurs when eddies are deformed and the profilers surface between the equivalent radii and 231 eddy edges.

\subsection{Isopycnal temperature anomalies in new-born eddies}

These depth-dependent $\theta / S$ profiles were first projected onto density-coordinates (here density refers to

234 potential density referenced to the sea-surface). Second, isopycnal $\theta^{\prime}$ and $S$ ', were inferred for each profile by

235 removing a local climatological profile representative of the large-scale background, also computed on density-

236 coordinates. In a similar way to Castelao et al. (2014) and Pegliasco et al. (2015), local climatological profiles (

$237 \bar{P}$ ), were obtained by weighted arithmetic means of all the available profiles $\left(\mathrm{P}_{\mathrm{i}}\right)$ acquired outside eddies, 238 within a radius of $200 \mathrm{~km}$ and separated by less than \pm 30 days (independently of the year) from the date of the 239 considered profile within the eddy (see Appendix A1). Selecting outside-eddy profiles collected no more than 30 240 days apart of the day of the year in which the eddy profile was collected, allows us to filter out unwanted $\theta / S$ 241 seasonal variations.

Vortices were then further classified into three main categories, depending on whether isopycnal $\theta^{\prime}$ were

243 i) significant (positive or negative) in the surface layer, extending from the surface to the base of the pycnocline,

244 ii) significant (positive or negative) in the subsurface layer below the base of the pycnocline, or iii) not 245 significant neither above nor below the pycnocline (see Appendix). The corresponding eddies are then qualified 246 as surface eddies, subsurface eddies or eddies with non-significant anomalies, respectively. Eddies having both 247 surface and subsurface significant isopycnal $\theta^{\prime}$ are considered as subsurface eddies. Note that the nature (surface 248 or subsurface) of the eddies is often defined based on kinematic properties such as geostrophic velocity (e.g. 249 Chaigneau et al., 2011; Pegliasco et al., 2015), vorticity (e.g. Assene et al., 2020), or Okubo-Weiss parameter 250 (e.g. Xu et al., 2019). Here in contrast, it is the vertical position of the significant isopycnal $\theta^{\prime}$ within the eddies 251 (above or below the pycnocline, respectively) that defines their nature (surface or subsurface).

252 In order to determine whether $\theta^{\prime}$ within eddies are significant or not, an isopycnal $\theta^{\prime}$ threshold was 253 defined in a $1^{\circ} \times 1^{\circ}$ longitude/latitude grid at seasonal scale, from Argo profiles that surfaced outside eddies (see 254 Appendix). Figures 3a-b show the annual mean of $\theta^{\prime}$ thresholds for the surface and subsurface layers, 
255 respectively. In general, a given $\theta^{\prime}$ profile acquired within an eddy is significant within the surface layer when 256 the square root of its quadratic mean values (see Eq. E4 in Appendix) integrated over the surface layer is greater 257 than $0.2-0.5^{\circ} \mathrm{C}$, except for some regions such as the frontal zone separating NAW from TSW, where $\theta^{\prime}$ threshold

258

259

260

261

262

263

264

265

266

267

268

269

270

271

272

273

274

275

276

277

278

279

reaches up to $0.8^{\circ} \mathrm{C}$ (Fig. 3a). In contrast, threshold values of $\theta^{\prime}$ within the subsurface layer are much lower and generally less than $0.3^{\circ} \mathrm{C}$. However, around the frontal zone along which the NEC is flowing, high $\theta^{\prime}$ threshold values are still noticed reaching up to $0.8^{\circ} \mathrm{C}$ (Fig. 3b). Note that a classification based on Student's t-tests was also performed and led to similar results at a $99 \%$ confidence level. For a given density, $\theta^{\prime}$ and $S^{\prime}$ are proportional and of the same sign, so that isopycnal maps of $\theta^{\prime}$ or $S^{\prime}$ are similar by definition. We thus hereinafter only focus on $\theta^{\prime}$ along isopycnal levels.

It is important to note that the ocean mixed layer is the site of intense ocean-atmosphere interactions that impact the mixed-layer properties from diurnal to inter-annual timescales (e.g. Guemas et al., 2011; Rugg and Foltz, 2016). For instance, diurnal variations of the solar heating can modify the daily mean SST by about a $0.3^{\circ}$ $0.5{ }^{\circ} \mathrm{C}$ (even greater for extreme events) in the TAO (e.g. Guemas et al., 2011; Gentemann et al., 2008; Wenegrat and McPhaden, 2015). Similarly, interannual variations of $\pm 0.5{ }^{\circ} \mathrm{C}$ are commonly observed in the TAO (e.g. Carton et al., 1996; Rugg and Foltz, 2016; Foltz et al., 2019). Thus, using Argo floats’ data within the mixed-layer induces relatively strong positive or negative $\theta / S$ anomalies that are not directly related to eddy dynamics and are difficult to interpret. Also, this high variability, can lead to a misclassification of eddies as the eddy signal in the mixed layer can be more variable than below the mixed layer. Thus in this study, surface eddies refer to eddies having significant $\theta^{\prime}$ between the base of the mixed layer to the base of the pycnocline.

\section{Results}

\subsection{Large-scale distribution of isopycnal temperature in the TAO}

In order to better understand the eddy signature on $\theta$ along isopycnal surfaces, we first briefly depict the large-scale water-mass $\theta$ distribution in the TAO along two isopycnal levels. As such, Fig. 4 presents the mean $\theta$ on $\sigma_{\theta}=25.5 \mathrm{~kg} \mathrm{~m}^{-3}$ and $\sigma_{\theta}=27.2 \mathrm{~kg} \mathrm{~m}^{-3}$ levels, obtained from Argo floats that surfaced outside eddies. These two particular $\sigma_{\theta}$ levels were retained because the maximum isopycnal $\theta^{\prime}$ within surface/subsurface eddies are 
mostly observed close to $\sigma_{\theta}=25.5 / 27.2 \mathrm{~kg} \mathrm{~m}^{-3}$ (see Fig. 7). On the shallower/lighter density-level, we can observe the noticeable warmer areas located within the subtropical gyres where NAW and SAW are located (Fig. 4a,d). On $\sigma_{\theta}=25.5 \mathrm{~kg} \mathrm{~m}^{-3}$ isopycnal, these water-masses $\theta$ ranges from 24 to $24.5{ }^{\circ} \mathrm{C}$ and 22 to $23{ }^{\circ} \mathrm{C}$, respectively (Fig. 4a,d). The along-isopycnal $\theta$ decreases equatorward from the gyre centers. In the eastern TAO, where EAW is originated, $\theta$ decreases to $\sim 20^{\circ} \mathrm{C}$ and $\mathrm{S}$ is lower than 36 (see also on Fig. $4 \mathrm{~d}$ ). Along the equator a relative warm core $\left(\theta \sim 22{ }^{\circ} \mathrm{C}\right)$ water-mass is advected eastward by the EUC (see Fig. 1 and Fig. 4a). The $\theta$ of this water-mass slightly decreases eastward and the water-mass is also characterized by relatively high salinities (not shown but see, e.g. Hormann and Brandt, 2007; Kolodziejczyk et al., 2009; Da-Allada et al., 2017). Finally, in the eastern boundary upwelling systems (Canary and Benguela), the mean $\theta$ is lower than $18{ }^{\circ} \mathrm{C}$ on $\sigma_{\theta}=25.5$ $\mathrm{kg} \mathrm{m}^{-3}$ due to the presence of the wind-forced coastal upwelling (Fig. 4a). The $\sigma_{\theta}=25.5 \mathrm{~kg} \mathrm{~m}^{-3}$ isopycnal layer deepens from $\sim 40 \mathrm{~m}$ in the eastern TAO to $\sim 140 \mathrm{~m}$ in the western TAO at latitudes of $\pm 20^{\circ}$ (black lines in Fig. 4a). The westward deepening of this isopycnal layer, which is associated with the lower part of the thermocline/pycnocline, is reduced along the equator where its depth varies from $50 \mathrm{~m}$ in the Gulf of Guinea to 100 m off Brazil.

On the $\sigma_{\theta}=25.5 \mathrm{~kg} \mathrm{~m}^{-3}\left(\sigma_{\theta}=27.2 \mathrm{~kg} \mathrm{~m}^{-3}\right.$, respectively) density layer, $\theta$ distribution shows a strong isopycnal $\theta$ front that separates the warmer and saltier NAW (NACW) from the colder and fresher EAW (SACW) and extends across the basin. East of $\sim 30^{\circ} \mathrm{W}$, this front is known as the Cape Verde Frontal Zone (CVFZ) (Zenk et al; 1991; Pérez-Rodriguez and Marrero-Diaz, 2001; Martínez-Marrero et al., 2008; Tiedemann et al., 2018), which is an active area of water-mass exchange associated with the formation of mesoscale eddies (e.g. Dadou et al., 1996; Schütte et al., 2016). However, we hereafter simply refer to the frontal zone to indicate the connection area between NACW and SACW. The NEC flows along this frontal zone, which is oriented southwestward from $20^{\circ} \mathrm{N}$ in the eastern TAO to $10^{\circ} \mathrm{N}$ in the western TAO (see also Fig. 1). This front is clearly visible down to $\sigma_{\theta}=27.2 \mathrm{~kg} \mathrm{~m}^{-3}$ (Fig. 4b) where it is more diffuse and the strongest $\theta$ gradients are observed southward, between $10^{\circ} \mathrm{N}$ and the equator. At this density level, the frontal zone is much more zonal, and NACW and SACW have typical $\theta$ of $10-11^{\circ} \mathrm{C}$ and 5-6 ${ }^{\circ} \mathrm{C}$, respectively (Fig. 4b,d). The mean depth of this 

isopycnal level is of $\sim 650 \mathrm{~m}$ in a large part of the TAO (black lines in Fig. 4b), and deepens to 750-900 m poleward of latitudes $\pm 20^{\circ}$.

Figure 4c presents the meridional $\theta$ section at $35^{\circ} \mathrm{W}$. Warmer waters with $\theta$ greater than $20{ }^{\circ} \mathrm{C}$ are located above the $\sigma_{\theta}=26.0 \mathrm{~kg} \mathrm{~m}^{-3}$ isopycnal layer and are associated with the previously described NAW, SAW and TSW. TSW is the warmest water-mass, with $\theta$ reaching $28{ }^{\circ} \mathrm{C}$ around the equator, but exhibits the lowest surface salinity of 35.5-36 (not shown) due to the excess of precipitation to evaporation in this area. Below the $\sigma_{\theta}$ $=26.0 \mathrm{~kg} \mathrm{~m}^{-3}$ density layer, there are the distinguishable warmer NACW and cooler SACW, separated by the $\theta$ front that becomes more diffuse below $\sigma_{\theta}=27.0 \mathrm{~kg} \mathrm{~m}^{-3}$.

$\theta / S$ diagrams and the main water-mass characteristics found in the TAO are depicted in Figure 4d. They were constructed from Argo floats that surfaced outside eddies within the northern, southern and eastern parts of the TAO (see boxes delimited in white in Fig 4a). This Figure confirms that TSW is much fresher and lighter than NAW or SAW and that NACW is warmer and saltier than SACW along isopycnal levels.

\subsection{Case studies of iso-depth versus isopycnal temperature anomalies in mesoscale eddies}

Estimates of $\theta$ and $S$ anomalies within an eddy can strongly differ whether we use a depth-coordinate system, where the local vertical displacement of isopycnal levels strongly impacts $\theta^{\prime} / S^{\prime}$, or a density-coordinate system. In order to better familiarize the reader with this concept, and to help the interpretation of the results described in the following Sections, we here describe $\theta^{\prime}$ observed in three individual mesoscale AEs at their generation sites, using both the depth- and density-coordinate systems. These three eddies (AE1, AE2 and AE3, respectively), detected by their ADT signature, were sampled by Argo floats in the western (AE1, Fig. 5a), central (AE2, Fig. 5b) and eastern parts (AE3, Fig. 5c) of the northern TAO. As typically observed in the TAO (Aguedjou et al., 2019), they have amplitudes of $\sim 2-4 \mathrm{~cm}$, radii of $\sim 70-100 \mathrm{~km}$, eddy kinetic energies of 85-100 $\mathrm{cm}^{2} \mathrm{~s}^{-2}$, and mean vorticities of $2-5 \times 10^{-5} \mathrm{~s}^{-1}$.

Although the 3 eddies were sampled by Argo floats within their core, AE1 and AE2 were sampled relatively close to their edge (Fig. 5a-b), whereas A3 was sampled in the vicinity of its center (Fig. 5c). As expected for AEs, $\theta^{\prime}$ observed using a depth-coordinate system are mainly positive for the 3 case-study eddies 
330 (Fig. 4d-f, magenta lines). Indeed, in depth-coordinates, the isopycnal deepening (rising, respectively) occurring 331 inside AEs (CEs), is the main driver of the observed positive (negative) $\theta^{\prime}$ (e.g. Assassi et al., 2016; Keppler et 332 al., 2018). The $\theta^{\prime}$ computed in depth-coordinates (Fig. 5d, magenta line) suggest that AE1 is a subsurface eddy with 334 a core extending from $100 \mathrm{~m}$ to $600 \mathrm{~m}$ depth. These anomalies are in part due to the observed $\sim 14 \mathrm{~m}$ deepening 335 of the subsurface isopycnal levels in AE1 (not shown). However, $\theta^{\prime}$ computed in density-coordinates (but plotted 336 in Fig 5d as a function of depth by associating each isopycnal level to its depth in the analyzed profile), are also 337 significantly positive in the $\sigma_{\theta} \sim 25.0-27.4 \mathrm{~kg} \mathrm{~m}^{-3}$ isopycnal layer located between 100 and $800 \mathrm{~m}$ depth, with 338 the maximum of $\sim 1^{\circ} \mathrm{C}$ reached slightly below $\sigma_{\theta}=27.0$ (Fig. $5 \mathrm{~d}$, red line). These positive isopycnal $\theta^{\prime}$ suggest 339 that diapycnal mixing or isopycnal advection from remote regions occurred during the formation of AE1. Fig. 5g 340 compares the $\theta / S$ diagrams for the Argo profile acquired inside the vortex (red) and the corresponding 341 climatological profile obtained from profiles outside eddies (green). It can be misleading since there exists 342 strong discrepancies between both profiles in low density ranges $\left(\sigma_{\theta}=24.0-25.5 \mathrm{~kg} \mathrm{~m}^{-3}\right)$ (Fig. 5d). However, 343 given the curvatures of isopycnal lines and of the vertical profiles, the strongest isopycnal eddy $\theta / S$ anomalies 344 are obtained in the subsurface layer containing NACW. Thus, for this particular AE1 case-study, $\theta^{\prime}$ are located in 345 subsurface and of the same sign (positive) for both coordinate systems.

346 In AE2, $\theta^{\prime}$ computed in depth-coordinates are positive between the surface and $250 \mathrm{~m}$ depth, and slightly 347 negative below $250 \mathrm{~m}$ (magenta line in Fig. 5e). The maximum anomaly is of $\sim 1.5{ }^{\circ} \mathrm{C}$ at $\sim 150 \mathrm{~m}$ depth, thus 348 AE2 is likely a surface eddy. In A2, these positive anomalies are associated with a deepening of the near-surface 349 isopycnal levels by $\sim 45 \mathrm{~m}$ (not shown). However, when computing $\theta^{\prime}$ in density-coordinates, AE2 is 350 characterized by negative $\theta^{\prime}$ in the surface layers reaching maximum negative anomalies of $-1.5^{\circ} \mathrm{C}$ for $\sigma_{\theta} \sim 26.0$ $351 \mathrm{~kg} \mathrm{~m}^{-3}$ at $\sim 170 \mathrm{~m}$ depth (red line in Fig. 5e). Again, these strong negative isopycnal $\theta^{\prime}$ suggest that AE2 contains 352 water from a remote region having distinct $\theta$-S characteristics, or that diapycnal mixing, likely associated with 353 air-sea fluxes, locally modified the thermohaline structure of the water column. AE2 clearly illustrates the 354 differences that can exist when using depth- versus density-coordinates to compute $\theta / S$ anomalies. 
Finally, in AE3, significant positive $\theta^{\prime}$ computed from depth-coordinates are observed from the surface to $200 \mathrm{~m}$ depth (Fig 5f) with maximum anomalies of $\sim 3.5{ }^{\circ} \mathrm{C}$ observed at $\sim 50 \mathrm{~m}$ depth. However, isopycnal $\theta^{\prime}$ show that this eddy does not contain significant water-mass anomalies relative to the background large-scale environment (Fig. 5f). Thus, $\theta^{\prime}$ observed in depth-coordinates only result from the local deepening of $\sim 12 \mathrm{~m}$ observed for the near-surface isopycnal layers (not shown). The isopycnal $\theta-S$ structure within AE3 is similar to the one usually observed in this region, with EAW and SACW in the surface and subsurface layers, respectively (Fig. 5i).

To summarize, based on 3 case-study AEs, we have shown that isopycnal $\theta^{\prime}$ anomalies can strongly differ from anomalies computed from the more commonly used depth-coordinate system. Obviously, similar conclusions hold for CEs that generally depict negative anomalies in depth-coordinate system, but that can show positive, negative or non-significant anomalies in density-coordinate system. These discrepancies are problematic, in particular for the estimates of anomalous heat or salt eddy contents, and their associated transports, which are commonly computed in depth-coordinates instead of using density-coordinates.

\subsection{Spatial distribution of isopycnal temperature anomalies in TAO new-born eddies}

Figure 6 shows the spatial distribution of surface and subsurface eddies, as well as eddies with nonsignificant anomalies at their generation sites in the TAO. These sampled new-born eddies have a mean amplitude, radius and lifespan of $\sim 3 \mathrm{~cm}, \sim 90 \mathrm{~km}$, and 150 days respectively. These mean values are associated with standard errors of $\sim 0.2 \mathrm{~cm}, \sim 2 \mathrm{~km}$ and $\sim 7$ days, respectively. A first striking result is that about half of the analyzed new-born AEs and CEs have a weak and non-significant isopycnal $\theta^{\prime}$ relatively to their local environment at their generation site, with their mean $\theta^{\prime}$ ranging mostly between $\pm 0.1^{\circ} \mathrm{C}$. They cover all areas and their spatial distribution is quite identical for AEs and CEs (Fig. 6e-f). Although not associated with significant isopycnal $\theta^{\prime}$, these eddies are associated with an average vertical displacement of $\pm 10 \mathrm{~m}$ of their isopycnal levels, similar to those observed in eddies with significant anomalies, which shows that both type of vortices are associated with dynamical signature of similar strength. Likewise, Chen et al., (2021) reported that eddies induce an average vertical displacement of the pycnocline of the same order of magnitude in tropical 
380

381

382

383

384

385

386

387

388

389

390

391

392

393

regions. As a result of the pycnocline vertical displacement, more than $90 \%$ of the new-born eddies that do not have significant isopycnal $\theta^{\prime}$ anomalies, exhibit significant $\theta^{\prime}$ in depth coordinates.

Thus, about half of the new-born eddies have significant $\theta^{\prime}$ anomalies at their generation sites (Fig. 6ad). Around $25 \%$ of these eddies are surface eddies whereas the remaining ( $75 \%)$ exhibit significant subsurface $\theta^{\prime}$. Note however, that $\sim 30 \%$ of these subsurface eddies also exhibit significant surface anomalies. The spatial distribution of AEs and CEs having significant isopycnal $\theta^{\prime}$ is almost similar in surface and subsurface layers (Fig. 6a-b and 6c-d). In the Southern Hemisphere, eddies with significant $\theta^{\prime}$ are very few and their maximum isopycnal $\theta^{\prime}\left( \pm 0.3^{\circ} \mathrm{C}\right.$ ) are observed around the Benguela upwelling system. In contrast, in the Northern Hemisphere, positive and negative $\theta^{\prime}$ are more similarly distributed with magnitude reaching up to $\pm 0.8^{\circ} \mathrm{C}$ along the frontal zone (Fig. 6a-b). Within the subsurface layer, AEs and CEs in the Northern Hemisphere are mostly characterized by negative and positive $\theta^{\prime}$, respectively, contrary to what is observed in the Southern Hemisphere (Fig. 6c-d). Again, this observed dominance of relatively cold AEs and warm CEs in subsurface is unusual when working in depth-coordinates where the sign of the anomalies is largely driven by the deepening/rising of isopycnal layers (see Section 3.2).

As described in Section 1, different mechanisms can create PV anomalies and generate eddies. However, depending on the involved mechanisms, the newly formed eddies either have isopycnal $\theta^{\prime} / S^{\prime}$ or not. Frictional mechanisms such as wind, lateral friction, or current shear, produce PV anomalies without creating isopycnal $\theta^{\prime} / S^{\prime}$. In contrast, the formation of PV anomalies by advection of water-masses with different characteristics or by diapycnal mixing is associated with isopycnal $\theta^{\prime} / S^{\prime}$. Thus, from the results obtained above, we can conclude that about $50 \%$ of the TAO eddies are likely formed by frictional mechanisms, whereas $50 \%$ are likely formed by diapycnal mixing or lateral advection of distinct water-masses. The majority of these new-born eddies have distinct $\theta^{\prime} / S^{\prime}$ in subsurface isopycnal layers with cold anomalies in AEs and warm anomalies for CEs in the Northern TAO. We decided to further study this area in the following section. 
Three sub-regions (R1 to R3, see Fig. 3-6) were defined according to their large-scale dynamics and

405

406

407

408

409

410

411

412

413

414

415

416

417

418 characteristics (Fig. 1, Fig. 3 and Fig. 4a-b). R1 extends along the frontal zone separating NACW from SACW (Fig. 3). The westward NEC which flows along the frontal zone is mainly fed by the eastern branch of the North Atlantic subtropical gyre but also by the northern branch of the Guinean Dome (Fig. 1). Further west, a part of the NEC retroflects cyclonically and feeds both the eastward North Equatorial Counter Current (NECC) and the eastward North Equatorial Undercurrent (NEUC) (Fig. 1a-b; e.g. Stramma and Schott, 1999; Bourlès et al. 1999a; Schott et al., 2004). The large-scale isopycnal $\theta$ distribution (Fig. 4) shows that the NEC flows along the frontal zone that separates relatively warm and salty waters of the North Atlantic subtropical gyre from cooler and fresher waters of the equatorial Atlantic Ocean (Fig. 4). This frontal zone is known to exhibit strong meanderings and eddy generations (e.g. Dadou et al., 1996; Shütte et al., 2016; Aguedjou et al., 2019). In R2, that extends from north-east Brazil to West Africa between $0^{\circ} \mathrm{N}$ and $10^{\circ} \mathrm{N}$ (Fig. 6), is found the zonal equatorial dynamics (Fig. 1). In this region, strong instabilities are frequently observed, in particular due to the horizontal shear between the nSEC and NECC (Fig. 1) (e.g. Weisberg and Weingartner, 1988; Kelly et al., 1995; Athié and Marin, 2008; Von Schuckmann et al., 2008, Aguedjou et al., 2019). Finally, subregion R3 includes the NBC retroflection (Fig. 1a-b), and is populated by relatively large and energetic eddies whose surface properties (size, amplitude, eddy kinetic energy) exhibit a strong seasonal variability (e.g. Aguedjou et al., 2019).

Figure 7 shows for these 3 subregions the mean vertical $\theta^{\prime}$ profiles inside new-born AEs and CEs using density-coordinates. As already discussed, the 3 sub-regions show a high number of non-significant anomaly profiles representing 40-60\% of observed eddies (Fig. 7, in black).

In R1, 75\% of the new-born eddies (AEs and CEs) having a significant $\theta^{\prime}$ are subsurface eddies (Fig. 7a,d). Surface AEs (solid lines in Fig. 7a) and CEs (solid lines in Fig. 7d) are characterized by average $\theta^{\prime}$ of $\pm 0.5-0.7^{\circ} \mathrm{C}$ between $\sigma_{\theta} \sim 25.5$ and $26.0 \mathrm{~kg} \mathrm{~m}^{-3}$. Subsurface AEs (dotted lines in Fig. 7a) and CEs (dotted lines in Fig. 7d) show maximum anomalies of $\pm 0.4{ }^{\circ} \mathrm{C}$ between $\sigma_{\theta} \sim 27 \mathrm{~kg} \mathrm{~m}^{-3}$ and $\sigma_{\theta} \sim 27.2 \mathrm{~kg} \mathrm{~m}^{-3}$ except subsurface cold CEs whose mean anomaly of $-0.5{ }^{\circ} \mathrm{C}$ is found between $\sigma_{\theta} \sim 26-26.5 \mathrm{~kg} \mathrm{~m}^{-3}$. Interestingly, around $73 \%(65 \%$, respectively) of these subsurface eddies are cold (warm) for AEs (CEs ). 
In R2, the surface eddies have strong anomalies of $\pm 0.5{ }^{\circ} \mathrm{C}$ just below the mixed layer, with the maximum located in the density range of the eastward NECC and the westward nSEC, which carry relatively warm and cold water, respectively (Fig. 1a and Fig. 4a). In the subsurface layer, similarly to what is observed in

R1 with a lower contrast with the surface layer, $\sim 65 \%$ of the CEs and $~ 70 \%$ of the AEs that present a significant (warm) anomalies and their maximum anomalies of $\sim \pm 0.3{ }^{\circ} \mathrm{C}$ are located at $\sigma_{\theta} \sim 27.2 \mathrm{~kg} \mathrm{~m}^{-3}$ (Fig. 7b,e).

floats. Moreover $\sim 55 \%$ of the AEs and $\sim 65 \%$ of the CEs sampled at their generation sites do not exhibit $\pm 1^{\circ} \mathrm{C}$ for CEs and $\sim \pm 0.7^{\circ} \mathrm{C}$ for AEs. Note, that the relatively small number of sampled eddies is due both to the very few number of Argo floats in R3 and to the reduced size of this area. AEs characterized by positive $\theta^{\prime}$ (red lines in Fig. 7c) show anomalies of $\sim 0.5{ }^{\circ} \mathrm{C}$, observed at $\sigma_{\theta}=26 \mathrm{~kg} \mathrm{~m}^{-3}$ for surface AEs, and $\sigma_{\theta}=26.8 \mathrm{~kg} \mathrm{~m}^{-3}$ for

subsurface AEs. Surface CEs predominantly show strong positive and negative anomalies that are maximum at $\sigma_{\theta}=25.5 \mathrm{~kg} \mathrm{~m}^{-3}$ (solid lines in Fig 7). In contrast, subsurface CEs mainly show positive $\theta^{\prime}$ of $0.5^{\circ} \mathrm{C}$ at the base of

443 the pycnocline or at $\sigma_{\theta} \sim 27 \mathrm{~kg} \mathrm{~m}^{-3}$ (dotted lines in Fig. 7f). Note that the 3 profiles classified as cold subsurface 444 CEs (blue dotted line in Fig. 7e) are characterized by strong positive anomalies between $\sigma_{\theta}=24.5 \mathrm{~kg} \mathrm{~m}^{-3}$ and $\sigma_{\theta}=$ $44526.7 \mathrm{~kg} \mathrm{~m}^{-3}$ and weak negative anomalies in deeper levels. Integrated in the water column, these deeper negative anomalies, that occupy a thicker layer, prevail and lead to a negative heat content anomaly that explains the classification of these profiles as cold subsurface CEs. In R3, the sampled new-born eddies were mainly formed on the eastern flank of the NBC retroflection (not shown), where relatively strong $\theta$ gradients exist. In the subsurface layer, isopycnal mixing of NACW and SACW takes place (eg. Kirchner et al., 2009).

\section{Qualitative analysis and discussion}

\subsection{Eddy vertical structure and potential implications for heat and salt transports}

The first striking result of our analysis, underlined in Fig. 6, is that about half of the analyzed eddies

453 have non-significant isopycnal $\theta^{\prime} / S^{\prime}$ at their generation site. The other half is highly dominated by subsurface 
454 eddies, with the strongest anomalies within the frontal zone in the Northern Hemisphere. The high number of

455 subsurface eddies obtained in our study have been also previously reported in different areas of the TAO, from both numerical simulations and in-situ data. For instance, in the Canary and Benguela upwelling systems, Pegliasco et al. (2015) estimated that subsurface eddies represent between 40 and $60 \%$ of the total number of eddies. Although their analysis was based on depth-coordinates and did not specifically focused on new-born eddies, these authors also showed that $40-60 \%$ of the sampled eddies do not have statistically significant $\theta^{\prime} / S^{\prime}$, in agreement with our results. Other studies also revealed the presence and persistence of subsurface structures in 461 various parts of the TAO, in the eastern and western boundary currents regions and in the subtropical gyres (e.g. 462 Schütte et al., 2016; Garraffo et al., 2003; Assene et al., 2020; Amores et al., 2017; Laxenaire et al., 2020). 463 However, as already discussed by Pegliasco et al. (2015), the high number of subsurface eddies could be 464 influenced by a slight sampling bias in Argo data. Indeed, Argo floats drift for 10 days at a nominal parking 465 depth of 500-1000 m and may therefore be preferentially trapped within subsurface eddies that reach these 466 depths.

In terms of tracer transport, our results would suggest that only half of the analyzed eddies (eddies with significant anomalies) might potentially contribute to the heat and salt transport within the basin. However, the 469 efficiency of the eddies to redistribute heat and salt through isopycnal advection from their formation region 470 strongly depends on the ambient $\theta / S$ characteristics at their dissipation sites and whether or not the trapped and 471 transported water-masses exhibit similar properties than the ambient ones. In the same way, other significant 472 processes such as diapycnal mixing along eddy trajectories, local eddy-stirring transport associated with the 473 lateral advection of large-scale thermohaline gradients (e.g. Sun et al., 2019) were not considered in our 474 estimation. Making a precise quantification of the eddy heat/salt transport exceeds the scope of this study. 475 However, it is important to recall that $\theta^{\prime} / S^{\prime}$ computed in depth-coordinates are largely influenced by the vertical displacement of isopycnal layers and do not reflect the net effect of eddy heat and salt contents that participate to 477 the redistribution of heat and salt in the global ocean. As such, isopycnal coordinates must be considered when 478 evaluating heat/salt transport by eddies. 
$481 \quad \frac{d}{d t} \vec{U}+\vec{f} \times \vec{U}=\frac{-\vec{\nabla} P}{\rho_{o}}-\vec{g} \frac{\rho}{\rho_{o}}+\vec{F}$

$\nabla(\vec{U})=0$

$\frac{d}{d t} \rho=\dot{\rho}$

484 where $\vec{U}=(u, v, w)$ is the velocity field, $\dot{f}$ is the Earth rotation vector, whose projection on the local vertical 485 axis defines the Coriolis parameter $f, \mathrm{P}$ is the pressure, $\rho$ the density of seawater and $\rho_{o}$ a mean density, $\vec{g}$ is the 486 gravity, $\frac{d}{d t} .=\partial_{t} .+u \partial_{x} .+v \partial_{y} .+w \partial_{z} \cdot$ is the Lagrangian derivative. Finally $\vec{F}$ and $\dot{\rho}$ are non-conservative terms respectively representing frictional effects (acting on momentum) and diapycnal mixing (acting on $\theta$ and/or S and finally modifying the particle potential density). As shown by Ertel (1942; see also Muller, 2006; Morel et al, 2019; Assene et al, 2020), we can define a generalized PV as:

$$
P V=-(\vec{\nabla} \times \vec{U}+\vec{f}) \cdot \vec{\nabla} Z(\rho)
$$

491 The traditional Ertel PV (obtained with $\mathrm{Z}(\rho)=\rho$ ) is dominated by the signature of the pycnocline, so that the 492 dynamical signal associated with isopycnal variations of PV is difficult to identify. In the following, we will use 493 the rescaled PV, as proposed in Delpech et al (2020) and Assene et al (2020), determined by choosing Z( $\left.\rho^{*}\right)=\mathrm{z}$ 494 for a specific location, where the density profile $\rho^{*}(\mathrm{z})$ is typical of the stratification of the area. The rescaled PV 495 is close to the quasi-geostrophic PV and scales as a vorticity with a reference PV value at rest close to $f$. Its 496 evolution equation is (see Muller, 2006):

$497 \quad \frac{\partial P V}{\partial t}=-\vec{\nabla} \cdot(\vec{U} \cdot P V+\vec{F} \cdot \vec{\nabla} Z(\rho)+(\vec{\nabla} \times \vec{U}+\vec{f}) \cdot \dot{Z}(\rho))$

498 This equation shows that PV anomalies can be formed by adiabatic displacement of water parcels in a 499 background PV gradient, frictional effects (associated with the forcing term $\vec{F}$ ) and diapycnal mixing 
500 (associated with the forcing terms $\dot{\rho}$ and $\dot{Z}(\rho)=\partial_{\rho} Z(\rho) \dot{\rho}$ ). We assume the modification of PV and $\theta$ takes

501 place in water masses of larger scale currents, and that vortices emerging from these currents keep similar

502 characteristics. Figure 8 qualitatively describes the expected effects of the identified three main mechanisms on

503 both the formation of PV and $\theta$ anomalies. First, since a water mass has specific isopycnal $\theta$ (and S) but

504 generally also PV signatures, the adiabatic displacement of a water mass to a region with different characteristics

505 potentially leads to the generation of eddies whose cores contain both PV and $\theta$ anomalies (Fig. 8 a-b). Second,

506 if both diapycnal fluxes $\dot{\rho}$ and frictional effects $\vec{F}$ can generate significant PV anomalies and potentially

507 strong eddies (Fig. 8c,e) (see Morel et al, 2019 and references therein), only diapycnal fluxes (induced by mixing

508 or atmospheric fluxes) are also associated with a modification of the $\theta / S$ properties and the generation of

509 isopycnal $\theta$ anomalies (Fig. 8c-d). It is interesting to notice that interior mixing (Fig. 8c-d) generates new

510 isopycnal characteristics (green patch in Fig. 8d) that necessarily remain in the convex envelope of the initial

511 background $\theta /$ S profile (light green area, see Penney et al., 2020), whereas atmospheric fluxes can substantially

512 alter the initial $\theta / \mathrm{S}$ properties of waters (red characteristics). In contrast, the frictional term acts on momentum

513 and thus on PV (Fig. 8e), but does not affect other tracers, so that no isopycnal $\theta$ anomalies are expected (Fig.

514 8f). To conclude, among the mechanisms that can lead to the formation of PV anomalies and eddies, friction is

515 the only one which does not create isopycnal $\theta$ anomalies.

516 If a rigorous evaluation of the PV production rate requires 3D fields, as explained in Assene et al (2020),

517 we can simplify Eq. (5) to estimate order of magnitudes for some terms. First, in a background PV gradient $\beta_{\mathrm{bg}}$,

518 isopycnal advection of water parcel will generate a PV anomaly

$519 \quad \delta \mathrm{PV}=\beta_{b g} \delta Y$

520 where $\delta Y$ represents the displacement of the fluid parcels along the PV gradient. Similarly, for this adiabatic 521 process, an isopycnal temperature anomaly is created with

$522 \delta \theta=\gamma_{b g} \delta Y$

523 where $\gamma_{b g}$ is the background temperature gradient (assumed to be in the same direction as the PV gradient for 524 simplicity). 


$$
\delta P V=-f \frac{\delta V}{V}
$$

529

where $V$ is the initial volume of the region undergoing mixing and $\delta V$ is its variation associated with mixing. In contrast, the evolution temperature, in a layer subject to mixing, depends on values of temperature in adjacent layers. It can be roughly estimated considering a volume $V$ of water in a layer, having an initial temperature $\theta_{\mathrm{i}}$ mixes with a volume $\delta V$ of an adjacent layer having a temperature $\theta_{\mathrm{a}}$. The modification of temperature in the initial layer is then:

$$
\delta \theta=\frac{\delta V}{V+\delta V}\left(\theta_{a}-\theta_{i}\right)
$$

Finally, the effect of friction is more complicated to evaluate, as it depends on details of the wind or bottom stress structure and stratification close to the boundary but it is known to yield important PV changes too (see Thomas, 2005; Morel et al, 2006; Benthuysen and Thomas, 2012; Gula et al, 20015, 2016), but the frictional forcing term does not modify temperature or salinity.

In order to analyze the effect of these processes, we computed a mean large-scale rescaled PV, calculated using Eq. (4), where $\vec{U}$ is the geostrophic velocity field computed from the World Ocean Atlas $\theta / S$ climatology (Locarnini et al., 2018; Zweng et al., 2018) with a level of no motion at $1000 \mathrm{~m}$ depth and where the reference profile to define $\mathrm{Z}(\rho)$ is chosen at $27^{\circ} \mathrm{W}, 7.5^{\circ} \mathrm{N}$, a dynamically less intense area corresponding also to a lower surface density. Figure 9a,c show the spatial distribution of the obtained rescaled PV, averaged within both a near-surface $\left(\sigma_{\theta}=25.75-26.5 \mathrm{~kg} \mathrm{~m}^{-3}\right)$ and a subsurface layer $\left(\sigma_{\theta}=26.9-27.4 \mathrm{~kg} \mathrm{~m}^{-3}\right)$. For each layer, the mean $\theta$ distribution is also shown by black contours (Fig. 9a,c).

In R1, a reservoir of relatively strong (weak, respectively) positive PV, associated with relatively cold (warm) water is observed on the southern (northern) edge of the thermal front in the surface layer (Fig. 9a). Thus in this layer, an isopycnal PV advection tends to generate either i) positive PV anomalies associated with negative $\theta^{\prime}$, 
549 leading to the formation of cold core surface CEs, or ii) negative PV anomalies associated with positive $\theta^{\prime}$, 550 leading to the generation of warm-core surface AEs. This PV/ $\theta$ relationship is better depicted in Fig. 9b. In 551 contrast, in R2, maximum positive PV are associated with warmer $\theta$, suggesting that isopycnal advection tends 552 to generate cold AEs and warm CEs in the surface layer of this region (Fig. 9a-b). In R3, as shown in Fig. 9b, the $553 \mathrm{PV} / \theta$ relationship is more complex and not strictly monotonic, suggesting that both warm and cold CEs and AEs 554 can be generated by isopycnal advection, although the general tendency is closer to the R2 region (cold AEs and warm CEs). From Figure 7, we indeed observed a higher number of cold AEs in the surface layer in R2 and R3, representing $\sim 66 \%$ of the significant surface eddies.

In R1, the background gradient of PV is $\beta_{\mathrm{bg}} \sim 2 \times 10^{-11} \mathrm{~m}^{-1} \mathrm{~s}^{-1}$, similar to the planetary beta (but note the gradient has opposite orientation here). The background gradient of temperature has the same direction and we can estimate $\gamma_{\mathrm{bg}} \sim 4 \times 10^{-6}{ }^{\circ} \mathrm{C} \mathrm{m}^{-1}$. From Eq. (6) and (7) we can thus estimate that to create an eddy with significant vorticity and PV anomaly, say $\delta \mathrm{PV} \sim \pm 10^{-5} \mathrm{~s}^{-1}$, by isopycnal advection, fluid parcels must be displaced by $\delta Y \sim$ $500 \mathrm{~km}$. This yields a temperature anomaly $\delta \theta \sim \pm 2{ }^{\circ} \mathrm{C}$ (positive for AEs and negative for CEs) which is 3 to 4 times the commonly observed anomalies in the area for these types of vortices. Thus, even for vortices whose PV and temperature anomalies are coherent with the background fields and could possibly be generated by isopycnal advection, other processes probably play a role in their generation. Evaluating the possible influence of diapycnal mixing of the near surface layer with the deeper subsurface one, Eq. (8) shows that a relative increase of volume $\delta V / V \sim 0.25$ is necessary to reach $\delta \mathrm{PV} \sim 1.10^{-5} \mathrm{~s}^{-1}$ (using $\mathrm{f} \sim 4.10^{-5} \mathrm{~s}^{-1}$ ). Taking a temperature difference of $\theta_{\mathrm{i}}-\theta_{\mathrm{a}} \sim 5^{\circ} \mathrm{C}$ between adjacent layers, we get $\delta \theta \sim 1{ }^{\circ} \mathrm{C}$, about 2 times the observed anomalies in the area, so atmospheric fluxes, or other processes, also probably have to be taken into account if diapycnal mixing is to explain the characteristics of observed eddy structures. As mentioned above it is difficult to evaluate the effect of a frictional term, but this mechanism creates PV anomalies without modifications of the isopycnal temperature, so it cannot explain alone the formation of eddies with significant temperature anomalies. We can conclude that in region R1, in the near surface layer, the PV and temperature structure of most eddies are probably determined by a combination of processes to explain the observed limited temperature anomalies while still leading to significant PV anomaly, and vorticity. 
$\mathrm{PV} / \theta$ relationships were also examined in the subsurface layer where we note a general northward increase of both the PV and $\theta$ (Fig. 9c). This distribution, as well as the main PV/ $\theta$ relationship shown in Fig. 9d, suggests that large-scale isopycnal advection leads to the formation of cold subsurface AEs and warm subsurface CEs in the TAO. Thus, isopycnal advection likely explains the dominance (65-80\% of the subsurface structures) of warm CEs and cold AEs observed from the Argo profiles in R1 and R2 (Fig. 7). For other eddies, similar analysis as proposed above can be performed and yield the same conclusions: a combination of processes is necessary to explain some of the observed eddy structures.

Note that the PV (and temperature) structure of eddies without significant isopycnal $\theta^{\prime} / S^{\prime}$ signature could be explained by frictional effects alone. Since they are present everywhere and represent about half the eddy population, this would suggest that wind and bottom stress are important mechanisms in the transformation of PV and creation of PV anomalies feeding the core of eddies in the TAO. Likewise, it is tempting to argue that friction could also explain the generation of PV anomalies for vortices exhibiting significant but unstructured $\theta^{\prime} / S^{\prime}$ signature. Note that even a constant wind (with weak Ekman pumping effects) is able to modify PV along a front (Thomas, 2005), a process that also leads to the destabilization of upwelling currents (Morel et al., 2006), strong modification of Ekman drift (Morel and Thomas, 2009) or the reinforcement of preexisting vortical structures (Holmes et al., 2014). However, this remains hypothetical, and specific combinations of advection and mixing can also be imagined to explain the observed structures. In fact, up to now, we did not find a way to evaluate the influence of friction in the transformation of PV from observations alone.

\subsection{Limitations of the study}

Diagnostics proposed in this study remain mostly qualitative, but, to our knowledge, it is the first attempt to analyze the importance of non-conservative effects in the generation of eddies and their associated $\theta / S$ and PV properties using only observations. The respective influence of the wind-stress and diapycnal mixing in the formation of vortices can serve as reference for realistic numerical models, for which these processes are parameterized. Given the reasonable number of eddies sampled in this study, the statistics calculated here are 
599

600

601

602

603

604

605

606

607

608

609

610

611

612

613

614

615

616

617

618

619

620

621

622

623

thought to be significant, but could obviously be refined in the future, when more observations become available.

Although our classification of significant versus non-significant $\theta^{\prime} / S^{\prime}$ is robust, it is important to point out some limitations of our diagnostics. First, the fact that eddies must be sampled at their generation ( $\pm 200 \mathrm{~km})$ sites strongly reduces the number of analyzed eddies.

Second, some of Argo profiles classified as outside eddy profiles might have sampled vortices which were not detectable by altimetry, as it has been highlighted by previous studies (e.g. Garraffo et 2003; Assene et al., 2020). The isopycnal $\theta$ climatology, obtained from profiles supposedly acquired outside eddies and used to evaluate eddy anomalies could be slightly spoiled by this effect. However, given the very large number of Argo profiles available, we believe this remains marginal.

Third, the exact location, relative to the eddy-centers, of Argo floats that surfaced within eddies were not considered when calculating the eddy $\theta^{\prime} / S^{\prime}$ (Fig. 2d). However, both theoretically and practically, Argo vertical profiles are on average acquired at a distance of $2 / 3$ of the equivalent eddy radius from the eddy center (e.g. Chaigneau and Pizarro, 2005; Pegliasco et al., 2015; Fig 2d). Although it may slightly impact our results, eddy cores can generally be considered as homogeneous in $\theta / S$ and the general discussion on the mechanisms involved in the generation of $\theta^{\prime} / S^{\prime}$, that shape the whole eddy structure from the eddy center to the eddy edge, remains valid.

Fourth, in Fig. 8 we assumed the PV and $\theta$ characteristics of eddies are determined by the properties of the water-mass forming their cores, so the generation process itself is adiabatic (such as baroclinic/barotropic instabilities, overshoot of a current at a cape, ...). Friction or mixing could act during the formation process, and we can even imagine that they directly create eddies. This is the case for the formation of eddies by local wind or bottom stress in the lee of islands (e.g. Pullen et al, 2008; Ioannou et al, 2020) or local convection (McWilliams, 1985). However, we think these events do not modify our interpretation as even at local scales advection, friction or mixing have the same qualitative effects on PV and $\theta^{\prime} / S^{\prime}$. One may also wonder how the complex eddy dynamics, characterized by frequently observed merging or splitting events, may influence our results. In 
624 our study, which focused on the eddy isopycnal structure close to the eddy generation sites, only splitting events

625 can be problematic because the eddy tracking algorithm considers that a new eddy is formed at the separation 626 site of the parent trajectory (e.g. Pegliasco et al., 2015; Laxenaire et al., 2018). However, among the 3700 new627 born eddies sampled by Argo floats in the TAO, only 590 (16\%) were associated with splitting events. 628 Excluding these eddies from the analyses did not alter the main results and conclusions drawn in this study. 629 However, this point could deserve special consideration in future investigations. Fifth, although satellite altimetry has been proven to be an efficient approach to identify and track 631 subsurface eddies (e.g. Ciani et al., 2017), the exact position of the birth location of subsurface eddies could be 632 slightly biased using altimeter data. Indeed, subsurface eddies do not necessarily have a clear signature on sea 633 surface height which prevents their identification in altimetry maps (e.g. Assene et al., 2020). Therefore, in our 634 study, some subsurface eddies could have been formed a few days/weeks before being detected by altimetry. 635 However, quantifying such biases is not possible without using three-dimensional numerical simulations (e.g. 636 Ciani et al., 2017), what is beyond the scope of the present study. Sixth, we argued that the effect of a frictional forcing term $\vec{F}$ (Eq. 1) on PV modification (Eq. 5) is difficult to assess. An additional difficulty is that frictional processes are generally also accompanied with diapycnal mixing. This is indeed generally the case for the wind stress, which can thus be expected to lead to modification of $\theta / S$ too. However, strong mixing is usually associated with convection generated by atmospheric

641 heat fluxes, or strong vertical shear in ocean currents. Without such strong preconditioning, diapycnal mixing 642 associated with the wind or bottom stress is expected to be weak, with $\theta^{\prime} / S^{\prime}$ remaining non-significant. Finally, Classification of eddies with significant vs non-significant temperature anomalies relies 644 on thresholds defined according to the local background variability and computed from Argo profiles that 645 surfaced outside eddies (Appendix A2). This variability represents the typical anomaly a particle can get when 646 displaced adiabatically over a typical vortex diameter. Thus a vortex whose core has an isopycnal $\theta^{\prime}$ below the 647 threshold must have gained its PV anomaly from processes that do not significantly alter the local water masses, 648 which is typical of friction. We used the $80^{\text {th }}$ percentile of this variability to define $\theta^{\prime}$ threshold, 649 since the isopycnal $\theta^{\prime}$ profiles are distinguishably different from those of eddies with significant $\theta^{\prime}$ (see Fig. 5d-f 
650

651

652

653

654

655

656

657

658

659

660

661

662

663

664

665

666

667

668

669

670

671

672

673

674

and 7). The number of identified eddies obviously depend on the choice of the percentile but the proportion of eddies with non-significant $\theta^{\prime}$ remains important as long as the chosen percentile remains reasonable (the proportion is still $40 \%$ when choosing the 70 th percentile, it reaches $70 \%$ when choosing the 90 th percentile). Likewise, results are also sensitive the confidence level chosen for the performed Student's t-test. Indeed, the more we decrease the confidence level of the Student's t-test, the more we increase the number of significant anomalies.

\section{Summary and perspectives}

Combining 18 years of satellite altimetry and $\theta / S$ data acquired by Argo floats in the TAO, we first showed that isopycnal $\theta^{\prime} / S^{\prime}$ can strongly differ from the ones obtained using depth-coordinates. Indeed, although $\mathrm{AE}$ (CE, respectively) mostly induced positive (negative) $\theta^{\prime} / S^{\prime}$ in depth-coordinates, both AE and CE can exhibit positive, negative or non-significant isopycnal $\theta^{\prime} / S^{\prime}$. We then focused on the vertical structure of eddies close to their generation site, to investigate the proportion and distribution of new-born eddies and found that half of the total analyzed new-born eddies in the TAO are characterized by non-significant $\theta^{\prime}$. In contrast, the second half, composed of $70 \%$ of subsurface, and of $30 \%$ of near-surface eddies, likely involve diapycnal mixing and/or lateral advection. As a consequence, in terms of tracer transport, our results would suggest that eddies that are generated with a significant $\theta^{\prime} / S^{\prime}$ mostly contribute to heat and salt transport in the TAO.

Refined diagnostics in three selected subregions in the northern TAO, were proposed. Along the frontal zone and in the northern equatorial subregions, the mean vertical structure of eddies is dominated by subsurface eddies with maximum anomalies reaching up to $\pm 0.5^{\circ} \mathrm{C}$ mostly found between $\sigma_{\theta}=27$ and $27.2 \mathrm{~kg} \mathrm{~m}^{-3}$ isopycnal layers. For these subsurface eddies, $\sim 75 \%$ of AEs exhibit a negative maximum $\theta^{\prime}$ whereas $\sim 65 \%$ of CEs maximum $\theta^{\prime}$ are positive. In the third subregion, within the NBC retroflection, results are questionable because of the reduced number of eddies sampled by Argo profiles at their generation sites.

We then proposed qualitative diagnostics based on the links between $\theta^{\prime} / S^{\prime}$ and PV anomalies under the influence of isopycnal advection, friction or diapycnal mixing. For eddies with significant anomalies, in the surface layer, PV/ $\theta$ relationships suggest that isopycnal water-mass advection can explain the generation of PV 
anomalies of cold core AEs observed in R2 and R3 areas. In contrast, in R1, isopycnal advection would

676

677

678

679

680

681

682

683

684

685

686

687

688

689

690

691

692

693

694

695

696

697

698

699

700 preferentially lead to the generation of cold core CEs and warm AEs. But observations show no preference in $\theta^{\prime}$. Thus isopycnal advection alone cannot explain our diagnostics and, to modify PV or $\theta^{\prime} / S^{\prime}$ structures of watermasses, other processes must be involved too. Both the wind-stress or diapycnal mixing can explain the observations and, even though we think friction is probably a key process to explain the observed characteristics, it seems difficult to prove that one process is more important than the other. Additional diagnostics can be imagined to further analyze the relative influence of each process. For instance, friction associated with the wind stress is probably sensitive to the position of the ITCZ in the studied region, leading to weaker stress in summer in R1, when the ITCZ migrates from the equator to this region. Preliminary analysis has shown that the production rate of eddies with non-significant $\theta^{\prime} / S^{\prime}$ drastically drops during summer, which could be a sign that friction is indeed a major mechanism for the generation of PV and vortices in this region. However, this drop also exists for some other vortex types, and the atmospheric heat fluxes also vary seasonally, so the interpretation is not easy and deeper analyses are necessary before concluding. In the subsurface-layer, watermass advection is also suggested to explain the formation of PV anomalies of warm (cold, respectively) core CEs (AEs) especially in R1 and R2, which indeed corresponds to the distribution dominantly observed.

As far as perspectives are concerned, this study provide a new diagnostics that can be useful for numerical models. As mentioned above, frictional and diapycnal mixing effects are parameterized in the models, and thus imperfectly represented. Given the qualitative link between these processes and the transformation of both PV and isopycnal $\theta^{\prime} / S^{\prime}$ fields, combined diagnostics involving isopycnal $\theta^{\prime} / S^{\prime}$ and vorticity of eddies is thus challenging for numerical results. In particular, since a major fraction of the observed eddies are associated with non-significant $\theta / S$ anomalies, frictional effects are thought to play a major role in the generation of their PV structure. Parameterizations of frictional effects are very difficult to evaluate and remain one of the Achille’s heel of circulation models at mesoscale. The proposed diagnostics can thus be very useful to compare different parameterizations. Further, it would be interesting to combine isopycnal $\theta / S$ structures with trajectories to infer the long term behavior of specific long-lived eddies, having been sampled at different times by Argo floats, to analyze the evolution of their heat/salt contents. The present general approach can also be applied to other 
701

702

703

704

705

706

707

708

709

710

\section{Appendix A: Determination of the significance of eddy temperature anomalies}

\section{A1. Weighted arithmetic means}

713

714

715 These local climatological profiles ( ${ }^{\bar{P}}$ ) were obtained by weighted arithmetic means of all the available profiles

$716\left(\mathrm{P}_{\mathrm{i}}\right)$ acquired outside eddies, within a radius of $200 \mathrm{~km}$ and separated by less than \pm 30 days (independently of the

717 year) from the date of the considered profile. Weighted arithmetic mean profiles ( $\bar{P}$ ) were computed by 718 equation E1:

719

$$
\bar{P}=\frac{1}{\sum \Omega_{i}\left(r_{i}, t_{i}\right)} \sum \Omega_{i}\left(r_{i}, t_{i}\right) \cdot P_{i}(\rho)
$$

720 where 721 the profile $\mathrm{P}_{\mathrm{i}}$ (outside eddies) from the considered profile (located inside the eddy): 
724 significantly alters $\bar{P}$ and the results presented in the study.

\section{A2. Temperature anomaly threshold}

In order to determine whether $\theta^{\prime} / S^{\prime}$ obtained within new-born eddies are significant or not, a climatology

727 of monthly isopycnal $\theta^{\prime}$ thresholds was computed on a $1^{\circ} \times 1^{\circ}$ longitude/latitude grid within surface and

728 subsurface layers. The surface layer extends from the base of the mixed layer to the base of the seasonal

729 pycnocline and the subsurface layer extends from the base of the pycnocline to the deepest isopycnal level (Fig.

730 A1). As in de Boyer Montégut et al. (2004), the mixed layer depth was defined as the depth where the density

731 increased by $0.03 \mathrm{~kg} \mathrm{~m}^{-3}$ from its value at $10 \mathrm{~m}$ depth. The reference depth was chosen at $10 \mathrm{~m}$ in order to avoid

732 the strong diurnal cycle that occurs in the first few meters of the ocean (de Boyer Montégut et al. 2004). The

733 base of the pycnocline was determined as the depth of fluid layer possessing one half of the maximum of the

734 squared buoyancy frequency (see Cheng and Hsu, 2014). The square buoyancy is defined as :

$735 \quad N^{2}=\frac{-g}{\rho} \frac{d \rho}{d z}$

736 where g, $\rho$ and z, are gravity, density, and depth, respectively.

737 Typically, the surface layer extends from $\sigma_{\theta} \sim 22 \mathrm{~kg} \mathrm{~m}^{-3}$ to $\sigma_{\theta} \sim 26 \mathrm{~kg} \mathrm{~m}^{-3}$ density layers in equatorial regions

$738\left(15^{\circ} \mathrm{N}-15^{\circ} \mathrm{S}\right)$, and from $\sigma_{\theta} \sim 25 \mathrm{~kg} \mathrm{~m}^{-3}$ to $\sigma_{\theta} \sim 26.5 \mathrm{~kg} \mathrm{~m}^{-3}$ at mid-latitudes $\left(15-30^{\circ}\right)$. In both areas, the subsurface

739 layer extend from the base of the surface layer to $\sigma_{\theta} \sim 27.5 \mathrm{~kg} \mathrm{~m}^{-3}$. In both the surface and subsurface layers

740 (Fig. $A_{1}$ ), the monthly climatological $\theta^{\prime}$ thresholds were computed at each grid point as follows: first, we

741 selected all the profiles within $200 \mathrm{~km}$ around the grid point, that surfaced outside eddies during the

742 corresponding month (regardless of the year). Second, we computed for each profile, the square root of the

743 quadratic mean of $\theta^{\prime}$ integrated over the layer thickness using equation E4.

$744 \quad M_{1}=\sqrt{\frac{1}{\sum h\left(\rho_{i}\right)} \cdot \sum h\left(\rho_{i}\right) \cdot\left(\theta^{\prime}\left(\rho_{i}\right)\right)^{2}}$ 
745 where $\mathrm{h}\left(\rho_{\mathrm{i}}\right)$ and $\theta^{\prime}\left(\rho_{\mathrm{i}}\right)$ are the thickness and the $\theta^{\prime}$ of the isopycnal layer $\rho_{\mathrm{i}}$. Third, we retained the $80^{\text {th }}$ percentile 746 of M1 values as the monthly $\theta^{\prime}$ threshold at the considered grid-point. This percentile was carefully chosen after 747 a sensitivity study and is considered as significantly different from noise. For accuracy reasons, at least 30

748 monthly profiles are required around the grid-point to compute the threshold. Figures A2 and A3 show the 749 monthly $\theta^{\prime}$ threshold distributions for the surface and subsurface layers. Note the relatively high $\theta^{\prime}$ threshold 750 within the frontal zone throughout the year in both layers. No strong seasonality was observed, except a slight 751 increase of the $\theta^{\prime}$ threshold value in February in the surface layer (Fig. A2).

752 Isopycnal $\theta^{\prime}$ observed in an eddy is considered as significant if its M1 values in surface and/or 753 subsurface are higher than the corresponding thresholds shown in Fig. A2-A3. In contrast if M1 values in the

754 eddy are lower than the corresponding thresholds in both the surface and subsurface layers, eddy anomalies are 755 classified as non-significant. When an anomaly is significant in a given layer, its sign is given by that of the 756 mean $\theta^{\prime}$ computed over the layer, as follow:

$757 \quad M_{2}=\frac{1}{\sum h\left(\rho_{i}\right)} \cdot \sum h\left(\rho_{i}\right) \cdot \theta^{\prime}\left(\rho_{i}\right)$

758 A similar method was used by Itoh and Yasuda (2010) to identify warm and cold eddies in the northwestern 759 Pacific ocean.

\section{A3. Mean eddy dynamic properties}

The mean evolution of the study eddy properties (amplitude, radius and kinetic energy), similarely to 762 what has been already observed by several observational (e.g. Pegliasco et al., 2015; Sun et al., 2018; Aguedjou 763 et al., 2019) and numerical (Kurian et al., 2011) studies, follows three main phases during eddy life-cycle: the 764 growth phase, the maturity phase and the dacaying phase (Fig A4a-c). The first 20-30\% of eddy lifespan 765 represents eddy growth phase during which eddy properties stongly increase, whereas the maturity phase 766 corresponds to a plateau-like evolution of the mean properties representing $20-30 \%$ to $80 \%$ of eddy lifespan. 
767 The decaying phase is the last phase of eddy life-cycle during which eddy properties decrease rapidely. During 768 the growth phase, TAO eddies travel on average over $200 \mathrm{~km}$ (Fig A4d).

\section{Acknowledgments:}

772 Studies (CNES) through the Ocean Surface Topography Science Team (OSTST) and supported by the French

773 National Institute of Sciences of the Universe (INSU/CNRS). This work is also a contribution to the junior team

774 “SAFUME” (JEAI-SAFUME) associated with the French National Research Institute for Development (IRD) 775 and to the EU H2020 TRIATLAS project under grant agreement 817578. M. Aguedjou was supported by a PhD 776 fellowship from the IRD and the Cultural Action and Cooperation Service (SCAC) of the French Embassy in 777 Benin.

Gridded altimetry data were produced by SSALTO/DUACS and distributed by the Copernicus Marine

779 Environment Monitoring Service (http://marine.copernicus.eu/) and Argo data were collected and made freely 780 available by the Coriolis project and programs that contribute to it (http://www.coriolis.eu.org).

782 greatly improved the quality of this study. 


\section{References}

Aguedjou, H. M. A., Dadou, I., Chaigneau, A., Morel, Y., \& Alory, G. (2019), Eddies in the Tropical Atlantic Ocean and their seasonal variability. Geophysical Research Letters, 46. https://doi.org/10.1029/2019GL083925

Akuetevi, C. Q. C. \& Wirth, A. (2015), Dynamics of turbulent western-boundary currents at low latitude in a shallow-water model, Ocean Sci., 11, 471-481, https://doi.org/10.5194/os-11-471-2015

Amores, A., Melnichenko, O. \& Maximenko, N. (2017), Coherent mesoscale eddies in the North Atlantic subtropical gyre: 3-D structure and transport with application to the salinity maximum, Journal of Geophysical Research: Oceans, 122(1), 23-41, doi:10.1002/2016jc012256.

Aristégui, J., Sangra, P., Hernandez Leon, S., Canton, M., Hernandez Guerra, A., \& Kerling, J. L. (1994), Island induced eddies in the Canary Islands. Deep Sea Research Part I: Oceanographic Research Papers, 41(10), 1509-1525. https://doi.org/10.1016/09670637 (94)90058 2

Assassi, C., Morel, Y., Vandermeirsch, F., Chaigneau, A., Pegliasco, C., Morrow, R., et al. (2016). An index to distinguish surface and subsurface intensified vortices from surface observations. Journal of Physical Oceanography, 46(8), 2529-2552. https://doi.org/10.1175/JPO-D-15-0122.1

Assene, F., Morel, Y., Delpech, A., Aguedjou, M., Jouanno, J., Cravatte, S., et al. (2020). From mixing to the large scale circulation: How the inverse cascade is involved in the formation of the subsurface currents in the Gulf of Guinea. Fluids, 5(3), 147. https://doi.org/10.3390/ fluids5030147

Athié, G \& Marin, F. (2008), Cross-equatorial structure and temporal modulation of intraseasonal variability at the surface of the tropical Atlantic Ocean, J. Geophys. Res., 113, C08020, doi :1029/2007JC004332.

Benthuysen, J. \& Thomas, L. (2012), Friction and Diapycnal Mixing at a Slope: Boundary Control of Potential Vorticity. J. Phys. Oceanogr. 42, 1509-1523.

Bourlès, B., Gouriou, Y., \& Chuchla, R. (1999a), On the circulation in the upper layer of the western equatorial Atlantic. Journal of Geophysical Research 104, 21,151-21,170. 
Carton, J., A., Cao X., Giese, B., S., \& da Silva, A., M. (1996), Decadal and interannual SST variability in the tropical Atlantic Ocean. Journal of Physical Oceanography 26: 1165-1175.

812

Chaigneau, A., Eldin, G., \& Dewitte, B. (2009), Eddy activity in the four major upwelling systems from satellite altimetry (1992-2007), Prog. Oceanogr., 83(1-4),117-123, doi:10.1016/j. Pocean.2009.07.012

Chaigneau, A., Gizolme, A., \& Grados, C. (2008), Mesoscale eddies off Peru in altimeter records: Identification algorithms and eddy spatio-temporal patterns, Prog. Oceanogr.,79(2-4), 106-119,doi:10.1016/j.pocean. 2008.10.013.

Chaigneau, A., Le Texier A., Eldin G., Grados C., \&Pizarro O. (2011), Vertical structure of mesoscale eddies in the eastern South Pacific Ocean: A composite analysis from altimetry and Argo profiling floats, Journal of Geophysical Research, 116(C11), doi:10.1029/2011jc007134.

Chaigneau, A., \& Pizarro, O. (2005), Eddy characteristics in the eastern South Pacific, J. Geophys. Res., 110, C06005, doi:10.1029/2004jc002815.

Charney, J. G., \& Stern, M. E. (1962), On the stability of internal baroclinic jets in a rotating atmosphere. Journal of the Atmospheric Sciences, 19(2), 159-172. $\underline{\text { https://doi.org/10.1175/1520 }}$ 0469(1962)019<0159:OTSOIB>2.0.CO;2

Chelton, D. B., Schlax, M. G., \& Samelson, R. M. (2011), Global observations of nonlinear mesoscale eddies, Progress in Oceanography, 91(2), 167-216, doi:10.1016/j.pocean.2011.01.002.

Chen, X., Li, H., Cao, C. Chen, G. (2021), Eddy-induced pycnocline depth displacement over the global ocean. Journal of Marine Systems, 221 (2021) 103577, https://doi.org/10.1016/j.jmarsys.2021.103577

Cheng, M.-H., \& Hsu J.R.-C. (2014), Effects of varying pycnocline thickness on interfacial wave generation and propagation, Ocean Engineering, 88, 34-45, doi:10.1016/j.oceaneng.2014.05.018.

Ciani, D., Carton, X., Aguiar, A., B., Peliz, A., Bashmachnikov, I., Ienna, F., Chapron, B., \&Santoleri, R. (2017), Surface signature of Mediterranean water eddies in a long-term high-resolution simulation, Deep Sea Research Part I: Oceanographic Research Papers, 130, 12-29, $\quad$ doi:10.1016/j.dsr.2017.10.001. 
836

837

838

839

840

841

842

843

844

845

846

847

848

849

850

851

852

853

854

855

856

857

858

859

860

D’Asaro, E. A. (1988), Generation of submesoscale vortices: A new mechanism. Journal of Geophysical Research: Oceans, 93 (C6), 6685-6693.

Da-Allada, Y. C., Jouanno, J., Gaillard, F., Kolodziejczyk, N., Maes, C., Reul, N., et al. (2017). Importance of the Equatorial Undercurrent on the sea surface salinity in the eastern equatorial Atlantic in boreal spring. Journal of Geophysical Research: Oceans, 122, 521-538. https://doi. org/10.1002/2016JC012342

Dadou I., Garçon, V., Andersen, V. , FlierI, G. R., \& Davis, C. S. (1996), Impact of the North Equatorial Current meandering on a pelagic ecosystem: A modeling approach, Journal of Marine Research, 54, 311-342

de Boyer Montégut, C., Madec, G., Fischer, A. S., Lazar, A., \& Iudicone, D. (2004), Mixed layer depth over the global ocean: An examination of profile data and a profile-based climatology, J. Geophys. Res., 109, C12003, doi:10.1029/2004JC002378.

Delpech, A., Cravatte, S., Marin, F., Morel, Y. Gronchi, E., \& Kestenare, E. (2020), Observed Tracer Fields Structuration by Middepth Zonal Jets in the Tropical Pacific. J. Phys. Oceanogr. 50, 281-304, doi:10.1175/JPO-D-19-0132.1.

Ducet N., Le Traon P.-Y., \& Reverdun, G. (2000), Global high-resolution mapping of ocean circulation from TOPEX/Poseidon and ERS-1 and -2, J. Geophys. Res. 105 (C8), 19,477-19,498.

Emery, W. J. (2003), Water Types and Water Masses, Ocean Circulation, pp. 1556-1567, Elsevier Sci., Amsterdam, Netherlands

Emery, W.J., \& Meincke, J. (1986), Global water masses: summary and review. Oceanol. Acta 9 (4). 344-383.

Ertel, H. (1942), On hydrodynamic eddy theorems. Physikalische Zeitschrift 43, 526-529.

Foltz, G. R., Brandt, P., Richter, I., Rodriguez-Fonseca, B., Hernandez, F., Dengler, M., et al. (2019), The Tropical Atlantic Observing System. Front. Mar. Sci. 6:206. doi: 10.3389/fmars.2019.00206

Foussard, A., Lapeyre G., \& Plougonven R. (2019), Response of Surface Wind Divergence to Mesoscale SST Anomalies under Different Wind Conditions, Journal of the Atmospheric Sciences, 76(7), 2065-2082, doi:10.1175/jas-d-18-0204.1.

Frenger, I., Gruber N. , Knutti R., \& Münnich M. (2013), Imprint of Southern Ocean eddies on winds, clouds 
and rainfall, Nature Geoscience, 6(8), 608-612, doi:10.1038/ngeo1863.

862

863

864

865

866

867

868

869

870

871

872

873

874

875

876

877

878

879

880

881

882

883

884

885

Garraffo, Z. D., Johns, W. E., Chassignet, E. P., \& Goni, G. J. (2003), North Brazil Current rings and transport of southern waters in a high resolution numerical simulation of the North Atlantic. In G. J. Goni \& P. Malanotte Rizzoli (Eds.), Interhemispheric Water exchange in the Atlantic Ocean, Elsevier Oceanographic Series 68 (pp. 375-409). Amsterdam: Elsevier.

Gaube, P., Mcgillicuddy, D. J., Chelton, D. B., Behrenfeld M. J., \&Strutton, P. G. (2014), Regional variations in the influence of mesoscale eddies on near-surface chlorophyll, Journal of Geophysical Research: Oceans, 119(12), 8195-8220, doi:10.1002/2014jc010111.

Gentemann, C. L., Minnett, P. J., Le Borgne, P., \& Merchant, C. J. (2008), Multi-satellite measurements of large diurnal warming events, Geophys. Res. L ett., 35, L2260 2, doi:10.1029/2008GL035730.

Guemas, V., Salas-Mélia, D., Kageyama, M., Giordani, H., \& Voldoire, A. (2011), Impact of the ocean mixed layer diurnal variations on the intraseasonal variability of Sea Surface Temperatures in the Atlantic Ocean. J. Climate 24 (12), 2889-2914, http://dx.doi.org/10.1175/2010JCLI3660.1

Gula, J., Molemaker M., \& McWilliams, J. (2015), Topographic vorticity generation, submesoscale instability and $\quad$ vortex street formation in the Gulf Stream. Geophys. Res. Lett. 42, 4054-4062.

Gula, J., Molemaker, M., \& McWilliams, J. (2016), Topographic generation of submesoscale centrifugal instability and energy dissipation. Nat. Commun. 7, 12811, doi:10.1038/ncomms12811.

Haynes, P., \& McIntyre, M. (1987), On the evolution of vorticity and potential vorticity in the presence of diabatic heating and frictional or other forces. J. Atmos. Sci. 44 (5), 828-841.

Haynes, P., McIntyre, M. (1990), On the conservation and impermeability theorems for potential vorticity. J. Atmos. Sci. 47 (16), 2021-2031.

Herbette S., Morel Y. \& Arhan M. (2004), Subduction of a Surface vortex under an outcropping front. J. Phys. Ocean. 34, pp. 1610-1627.

Herbette S., Morel Y. \& Arhan M. (2003), Erosion of a surface vortex by a seamount. J. Phys. Oceanogr. 33, $1664-1679$ 
886

887

888

889

890

891

892

893

894

895

896

897

898

899

900

901

902

903

904

905

906

907

908

909

910

Holmes, R.M., \& Thomas, L.N. (2016), Modulation of Tropical Instability Wave Intensity by Equatorial Kelvin Waves. $\quad$ J. Phys. Oceanogr. 46, 2623-2643, doi:10.1175/JPO-D-16-0064.1.

Holmes, R. M., Thomas, L.N., Thompson, L., \& Darr, D. (2014), Potential Vorticity Dynamics of Tropical Instability ～Vortices. J. Phys. Oceanogr. 44, 995-1011, doi:10.1175/JPO-D-13-0157.1.

Hormann, V., \& Brandt P. (2007), Atlantic equatorial undercurrent and associated cold tongue variability, $J$. Geophys. Res., 112, C06017, doi:10.1029/2006JC003931.

Hoskins, B.J., McIntyre, M. E., \& Robertson, A.W. (1985), On the use and significance of isentropic potential vorticity maps. Q. J. R. Met. Soc. 470, 877-946.

Itoh, S., \& I. Yasuda (2010), Water Mass Structure of Warm and Cold Anticyclonic Eddies in the Western Boundary Region of the Subarctic North Pacific, Journal of Physical Oceanography, 40(12), 26242642, doi:10.1175/2010jpo4475.1.

Ioannou A., Stegner A., Dumas F., \& Le Vu B. (2020), Three-Dimensional Evolution of Mesoscale Anticyclones in the Lee of Crete, Frontiers in Marine Science, 7, 1019, doi:10.3389/fmars.2020.609156

Kelly, B., Meyers S. \& O’Brien J. (1995), On a generating mechanism for Yanai waves and the 25-day oscillation, J. Geophys. Res., 100, 10,589 - 10,612.

Keppler, L., Cravatte S., Chaigneau A., Pegliasco C., Gourdeau L., \& Singh A. (2018), Observed Characteristics and Vertical Structure of Mesoscale Eddies in the Southwest Tropical Pacific, Journal of Geophysical Research: Oceans, 123(4), 2731-2756, doi:10.1002/2017jc013712.

Kirchner, K., Rhein M., Hüttl-Kabus S. \& Böning C. W. (2009), On the spreading of South Atlantic Water into the northern hemisphere. J. Geophys. Res., 114, C05019, doi:10.1029/ 2008JC005165

Kolodziejczyk, N., Bourlès B., Marin F., Grelet J. \& Chuchla R. (2009), Seasonal variability of the Equatorial Undercurrent at $10^{\circ} \mathrm{W}$ as inferred from recent in situ observations, J. Geophys. Res., 114, C06014, doi:10.1029/2008JC004976.

Kurian, J., F. Colas, X. Capet, J. C. McWilliams, and D. B. Chelton (2011), Eddy properties in the California Current System, J. Geophys. Res., 116, C08027, doi:10.1029/2010JC006895. 
911 Laxenaire, R., Speich S., Blanke B., Chaigneau, A., Pegliasco C., \& Stegner A. (2018), Anticyclonic Eddies Connecting the Western Boundaries of Indian and Atlantic Oceans, Journal of Geophysical Research: Oceans, 123(11), 7651-7677, doi:10.1029/2018jc014270.

914 Laxenaire, R., Speich, S., \& Stegner, A. (2020), Agulhas ring heat content and transport in the South Atlantic 915 estimated by combining satellite altimetry and Argo profiling floats data. Journal of Geophysical

\section{7}

918

919 920

921 922

923

924 Research: Oceans, 125, e2019JC015511. https:// doi.org/10.1029/2019JC015511

Le Traon P.-Y., Nadal, F., \& Ducet, N. (1998), An Improved Mapping Method of Multisatellite Altimeter Data, J. Atmos. Oceanic Technol. 15, 522-534

Liu, M. \& Tanhua, T. (2019), Characteristics of Water Masses in the Atlantic Ocean based on GLODAPv2 data, Ocean Sci. Discuss., https://doi.org/10.5194/os-2018-139, in review.

Locarnini, R. A., Mishonov, A. V., Baranova, O. K. et al. (2018), World Ocean Atlas 2018, Volume 1: Temperature. A. Mishonov Technical Ed.; NOAA Atlas NESDIS 81, 52 pp.

Martínez-Marrero, A., Rodríguez-Santana A., Hernández-Guerra A., Fraile-Nuez, E., López-Laatzen, F., VélezBelchí, P., \& Parrilla, G. (2008), Distribution of water masses and diapycnal mixing in the Cape Verde Frontal Zone, Geophys. Res. Lett., 35, L07609, doi:10.1029/ 2008GL033229

McGillicuddy, D. J. (2016), Mechanisms of Physical-Biological-Biogeochemical Interaction at the Oceanic Mesoscale, Annual Review of Marine Science, 8(1), 125-159, doi:10.1146/annurev-marine-010814015606.

McWilliams, G. \& Flierl, R. (1979), On the evolution of isolated, nonlinear vortices. J. Phys. Oceanogr., 9, 1155-1182.

McWilliams, J. C. (1985), Submesoscale, coherent vortices in the ocean, Rev. Geophys., 23( 2), 165182, doi:10.1029/RG023i002p00165

Morel, Y. G., \& McWilliams, J.C., (1997), Evolution of isolated interior vortices in the ocean, J. Phys. Oceanogr. 27, 727-748.

Morel, Y., Gula J., \& Ponte A. (2019), Potential vorticity diagnostics based on balances between volume integral 
and boundary conditions, Ocean Modelling, 138, 23-35, doi:10.1016/j.ocemod.2019.04.004.

937

938

939

940

941

942

943

944

945

946

Morel, Y., \& McWilliams J. (2001), Effects of isopycnal and diapycnal mixing on the stability of oceanic currents. J. Phys. Oceanogr., 31, 2280-2296.

Morel, Y., Darr D., \& Tailandier, C., (2006), Possible sources driving the potential vorticity structure and longwave instability of coastal upwelling and downwelling currents. J. Phys. Ocean. 36, 875-896.

Morel, Y., \& Thomas, L. (2009), Ekman drift and vortical structures. Ocean Model. 27, 185-197

Morvan, M., L'Hégaret, P., Carton, X., Gula, J., Vic, C., de Marez, C., et al. (2019). The life cycle of submesoscale eddies generated by topographic interactions. Ocean Science, 15(6), 1531-1543. https://doi.org/10.5194/os-15-1531-2019

Muller, P. (2006), The Equations of Oceanic Motions; Cambridge University Press: Cambridge, UK.

Onken, R., \& Klein, B. (1991), A model of baroclinic instability and waves between ventilated gyre and the shadow zone of the North Atlantic Ocean, J. Phys. Oceanogr., 21, 53 - 67.

Pegliasco, C., Chaigneau, A., \& Morrow, R. (2015), Main eddy vertical structures observed in the four major Eastern boundary upwelling systems: J. Geophys. Res. Oceans, 120, 6008- 6033.

Penney, J., Morel, Y., Haynes, P., Auclair, F., \& Nguyen, C. (2020), Diapycnal mixing of passive tracers by Kelvin-Helmholtz instabilities. Journal of Fluid Mechanics, 900, A26. doi:10.1017/jfm.2020.483

Pérez-Rodriguez, P., Pelegri, J. L., \& Marrero-Diaz, A. (2001), Dynamical characteristics of the Cape Verde frontal zone, Sci. Mar., 65, 241-250.

Pujol, M.-I., Faugère, Y., Taburet, G., Dupuy, S., Pelloquin, C., Ablain, M., \& Picot, N. (2016). DUACS DT2014: The new multi-mission altimeter data set reprocessed over 20 years. Ocean Science, 12(5), 1067-1090. https://doi.org/10.5194/os-12-1067-2016

Pullen, J., Doyle, J. D., May, P., Chavanne, C., Flament, P., \& Arnone, R. A. (2008), Monsoon surges trigger oceanic eddy formation and propagation in the lee of the Philippine Islands. Geophys. Res. Lett. 35:L07604. doi: 10.1029/2007GL033109

Renault, L., Marchesiello, P., Masson, S., \& McWilliams, J. C. (2019), Remarkable control of western boundary currents by Eddy Killing, a mechanical air-sea coupling process. Geophysical Research Letters, 46. 
963 Rugg, A., Foltz, G.R., \& Perez, R.C. (2016), Role of mixed layer dynamics in tropical North Atlantic interannual 964 sea surface temperature variability. J. Clim. 29 (22), 8083-8101.

965 Saenko, O. A., Yang, D., \& Gregory, J. M. (2018), Impact of mesoscale eddy transfer on heat uptake in an 966 eddy parameterizing ocean model. Journal of Climate, 31(20), 8589-8606. https://doi.org/10.1175/jcli-d-18-0186.1

968 Schuckmann, K. V., Brandt, P., \& Eden, C. (2008), Generation of tropical instability waves in the Atlantic 969 Ocean, Journal of Geophysical Research, 113(C8), doi:10.1029/2007jc004712.

970 Schütte, F., Brandt, P., \& Karstensen, J. (2016), Occurrence and characteristics of mesoscale eddies in the 971 tropical northeastern Atlantic Ocean, Ocean Science, 12(3), 663-685, doi:10.5194/os-12-663-2016.

972 Seo, H., Miller, A., \& Norris, J. (2016), Eddy-wind interaction in the California current system: Dynamics and 973 impacts, J. Phys. Oceanogr., 46, 439-459, doi:10.1175/JPO-D-15-0086.1.

974 Snowden, D., \& Molinari, R. (2003), Subtropical cells in the Atlantic Ocean: An observational summary, in 975 Interhemispheric Water Exchange in the Atlantic Ocean, edited by G. Goni and P. Malanotte-Rizzoli, $976 \quad$ Elsevier Oceanogr. Ser., 68, $287-312$.

977 Sprintall, J., \& Tomczak, M. (1993), On the formation of central water in the southern hemisphere. Deep978 Sea $\quad$ Research 40, 827-848.

979 Stramma, L., \& Schott, F. (1999), The mean flow field of the tropical Atlantic Ocean, Deep Sea Res. II, 46, $980 \quad 279-303$.

981 Stramma, L., Juttl S., \& Schafstall, J. (2005a), Water masses and currents in the upper tropical northeast 982 Atlantic off northwest Africa, J. Geophys. Res., 110, C12006, doi:10.1029/2005JC002939.

983 Stramma, L., M. Rhein, Brandt, P., Dengler, M., Böning, C. \& Walter, M. (2005b), Upper ocean circulation in 984 the western tropical Atlantic in boreal fall 2000, Deep Sea Res., I, (52), 221 - 240. 
985 Sun, B., Liu, C. \& Wang, F. (2019), Global meridional eddy heat transport inferred from Argo and altimetry observations. Sci Rep 9, 1345. https://doi.org/10.1038/s41598-018-38069-2

Sun, W., Dong, C., Tan, W., Liu, Y., He, Y., \& Wang, J. (2018), Vertical structure anomalies of oceanic eddies and eddy-induced transports in the South China Sea. Remote Sens. 10, 795. https://doi.org/10.3390/rs10050795

990 991

992 993

994 995

996 997 998 999 1000 1001 1002 1003 1004 1005 1006 1007 1008

Sutyrin G. G. \& Flierl, G. R. (1994), Intense vortex motion on the beta-plane: development of the beta-gyres, J. Atmos. Sci. 51, 773-790

Sutyrin G.G. \& Morel, Y.G. (1997), Intense vortex motion in a stratified fluid on the beta-plane: an analytical theory and its validation, J. Fluid Mech. 336, 203-220.

Sverdrup, H.U., Johnson, M.W., \& Fleming, R.H. (1942), The Oceans. Their Physics, Chemistry, and General Biology. Prentice-Hall, $\quad$ Englewood Cliffs, NJ, 1087 pp.

Thomas, L.N. (2005), Destruction of Potential Vorticity by Winds. J. Phys. Oceanogr., 35, 2457-2466, doi:10.1175/JPO2830.1.

Thomas, M. D., \& Zhai, X. (2013), Eddy-induced variability of the meridional overturning circulation in a model of the North Atlantic, Geophys. Res. Lett., 40, 2742-2747, doi:10.1002/grl.50532

Tiedemann, M., Fock, H. O., Döring, J., Badji, L. B. \& Möllmann, C. (2018), Water masses and oceanic eddy regulation of larval fish assemblages along the Cape Verde frontal zone. J. Mar. Syst., 183, 42-55.

Tomczak, M., \& Godfrey, J.S. (1994), Regional Oceanography: An Introduction. Elsevier, Oxford, 422 pp.

Tsuchiya, M., Talley, L. D., \& McCartney, M. S. (1992), An eastern Atlantic section from Iceland southward across the equator, Deep Sea Res., 39, 1885 - 1917

Urbano, D. F., De Almeida, R. A. F., \& Nobre, P. (2008), Equatorial Undercurrent and North Equatorial Countercurrent at $38^{\circ} \mathrm{W}$ : A new perspective from direct velocity data. J. Geophys. Res., 113, C04041, doi:10.1029/2007JC004215

Vandermeirsch, F., Morel, Y., \& Sutyrin, G. (2001), The net advective effect of a vertically sheared current on a 
1009 coherent vortex. J. Phys. Oceanogr., 31, 2210-2225.

1010 Villas Bôas, A. B., Sato, O. T., Chaigneau, A. \& Castelão, G. P. (2015), The signature of mesoscale eddies on

1011 the air sea turbulent heat fluxes in the South Atlantic Ocean, Geophysical Research Letters, 42(6), 18561012 1862, doi:10.1002/2015gl063105.

1013 Wang, C. (2005), Subthermocline tropical cells and equatorial subsurface countercurrents. Deep Sea Res. Part 1014 Oceanogr. Res. Pap. 52, 123-135, doi:10.1016/j.dsr.2004.08.009.

1015 Weisberg R. H., \& Weingartner, T. J. (1988), Instability waves in the equatorial Atlantic Ocean, Journal of $1016 \quad$ Physical Oceanography, 18, pp. 1641-1657

1017 Wenegrat, J. O., \& McPhaden, M. J. (2015), Dynamics of the surface layer diurnal cycle in the equatorial 1018 Atlantic Ocean (0, 23 W). Journal of Geophysical Research: Oceans, 120, 563-581. 1019 https://doi.org/10.1002/2014JC010504

1020 Wilson, W., Johns, E., \& Molinari, R. (1994), Upper layer circulation in the western tropical North Atlantic 1021 Ocean during August 1989, J. Geophys. Res., 99, 22,513 - 22,523.

1022 Xu, A., Yu, F. \& Nan, F. (2019), Study of subsurface eddy properties in northwestern Pacific Ocean based on an 1023 eddy- resolving OGCM. Ocean Dynamics 69, 463-474. https://doi.org/10.1007/s10236-019- 01255-5

1024 Zenk, W., B. Klein, \& Schröder, M. (1991), Cape Verde Frontal Zone, Deep Sea Res., 38, suppl. 1, S505-S530

1025 Zweng, M. M., Reagan, J. R., Seidov, D. et al. (2018), World Ocean Atlas 2018, Volume 2: Salinity. A. 1026 Mishonov Technical Ed.; NOAA Atlas NESDIS 82, 50 pp. 


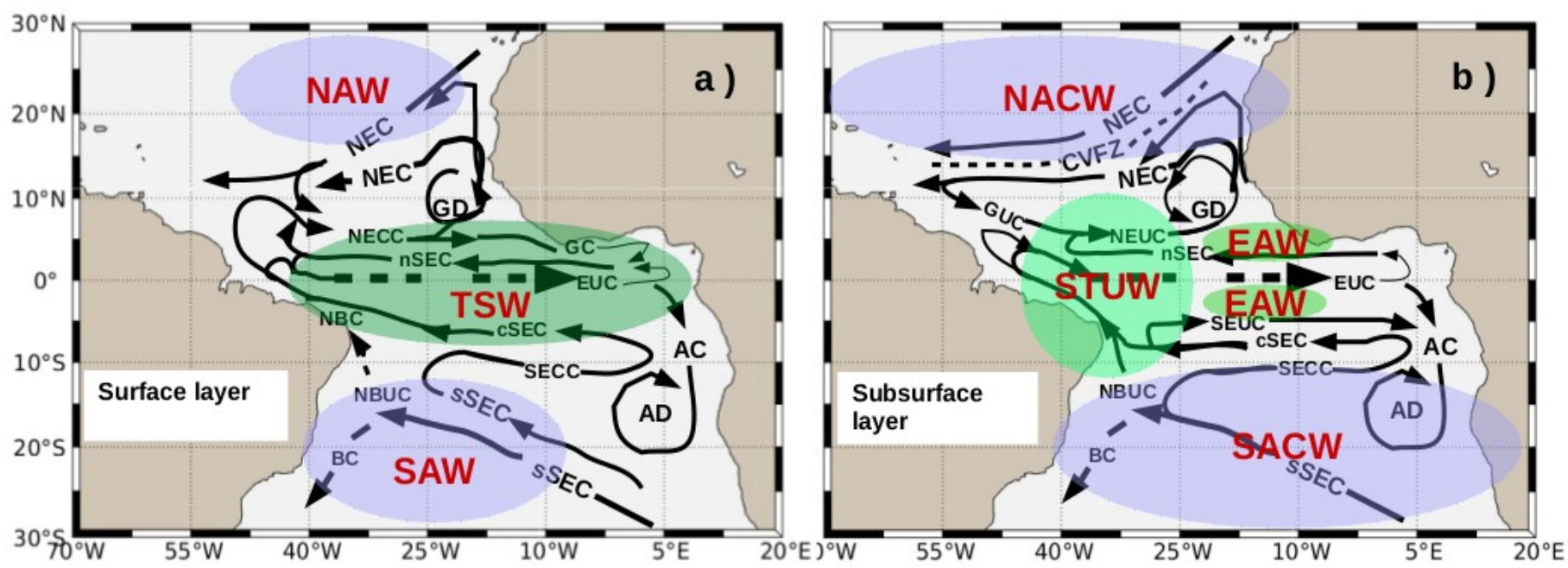

1032 Figure 1. Schematic view of a) surface and b) subsurface circulation in the TAO (adapted from Stramma and 1033 Schott, 1999). Main currents are: Brazil Current (BC), North Brazil Current (NBC), North Brazil Undercurrent 1034 (NBUC), North Equatorial Current (NEC), North Equatorial Countercurrent (NECC), northern, central and 1035 southern branches of South Equatorial Current (nSEC, cSEC, sSEC), Guinea Current (GC), Angola Current 1036 (AC), Angola Dome (AD), Guinea Dome (GD), South Equatorial Countercurrent (SECC), Equatorial 1037 Undercurrent (EUC), North Equatorial Undercurrent (NEUC), South Equatorial Undercurrent (SEUC), Guiana 1038 Undercurrent (GUC). Tropical surface water-masses and central water-masses are also indicated: Tropical 1039 Surface water (TSW), Northern, southern and eastern tropical Atlantic water (NAW, SAW, EAW), Subtropical 1040 Underwater (STUW), North and South Atlantic Central Water (NACW, SACW). 

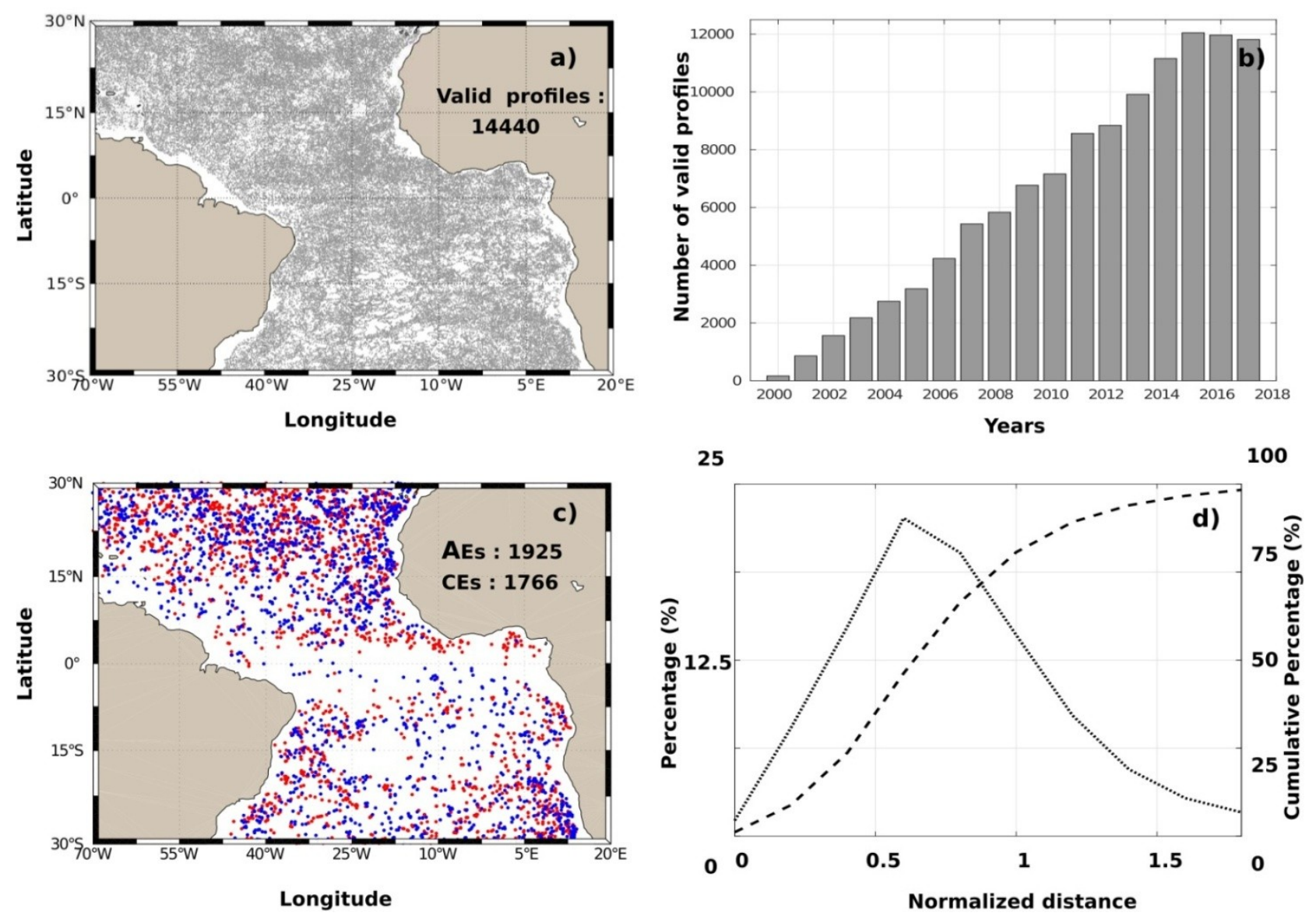

1046 Figure 2. Spatio-temporal distribution of Argo floats in the TAO: a) Spatial and b) temporal distribution of valid 1047 Argo profiles, c) spatial distribution of Argo profiles that surfaced within anticyclonic eddies (AEs, red dots) and 1048 cyclonic eddies (CEs, blue dots), d) Histogram (dotted line, left axis) and cumulative distribution (dashed line, 1049 right axis) of the normalized radial distance of Argo floats to eddy centers. Normalized distances were obtained 1050 dividing the distance (in $\mathrm{km}$ ) by the equivalent eddy radius. 


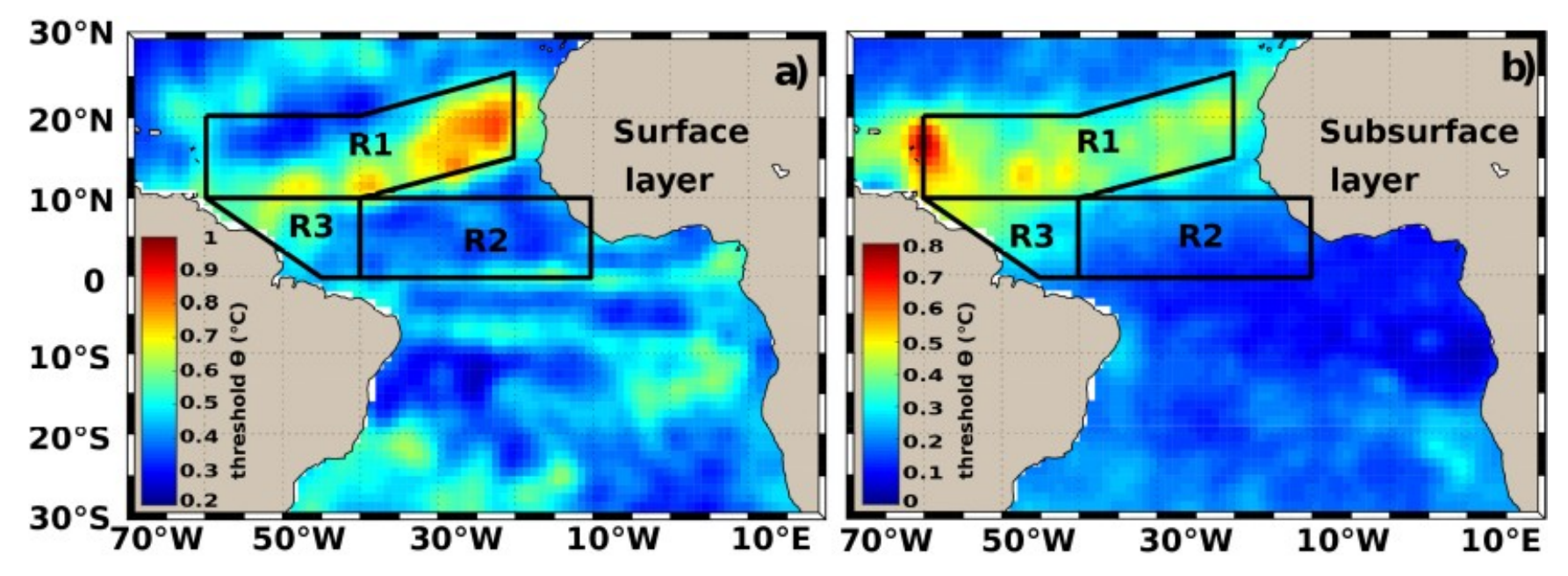

1054 Figure 3. Annual mean of the isopycnal temperature anomaly threshold within the a) surface and b) subsurface 1055 layers. Three dynamically different subregions (R1, R2, R3) used in this study are delimited in black (see in-text 1056 description of these regions). Surface layer extends from the mixed layer depth to the base of the pycnocline, 1057 whereas subsurface layer extends below the pycnocline to $1000 \mathrm{~m}$ depth. 1058 1059 1060 

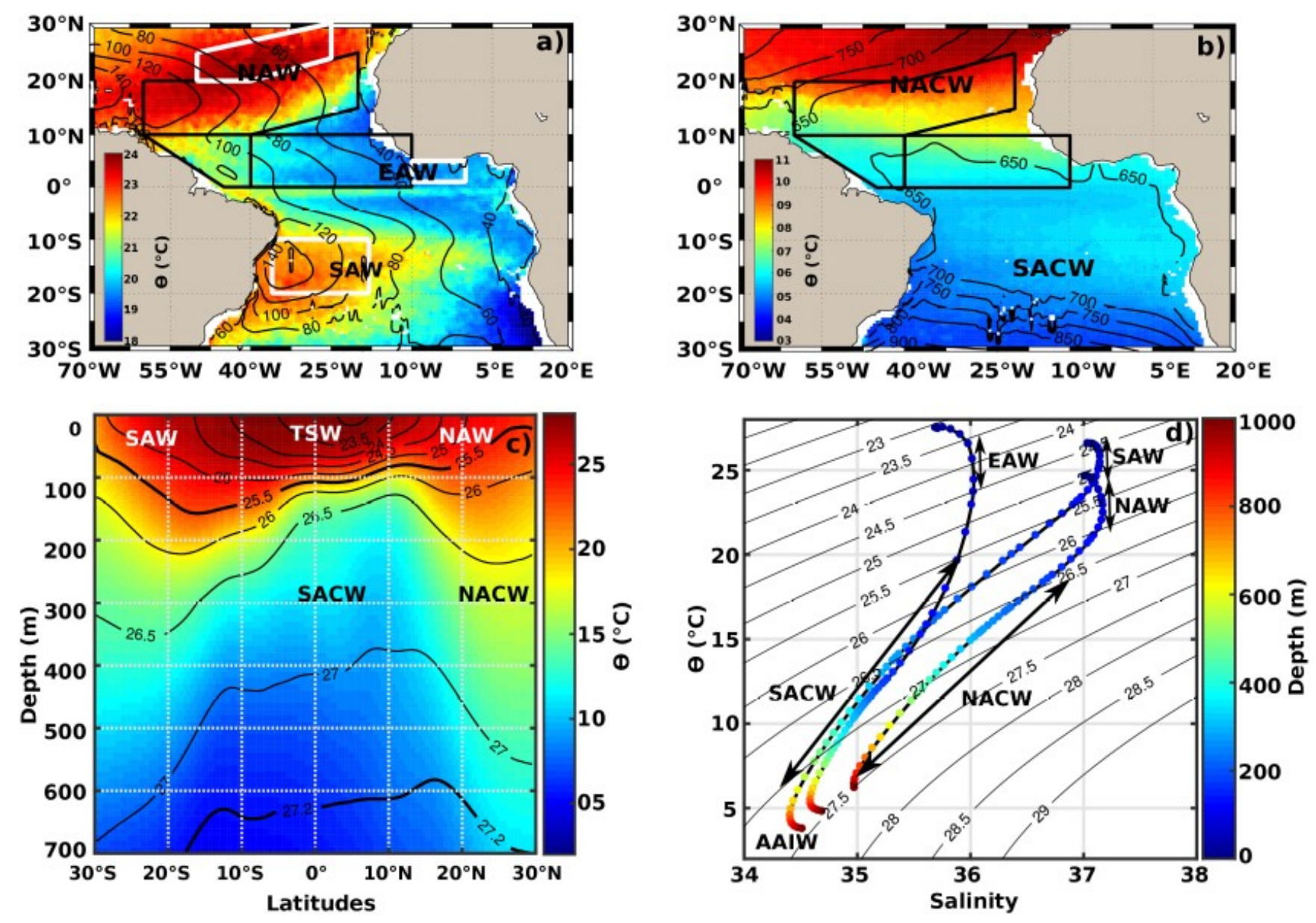

1062 Figure 4. Large-scale temperature distribution (in ${ }^{\circ} \mathrm{C}$ ) on a) $\sigma_{\theta}=25.5 \mathrm{~kg} \mathrm{~m}^{-3}$ and b) $\sigma_{\theta}=27.2 \mathrm{~kg} \mathrm{~m}^{-3}$. Black 1063 contours correspond to the depth (in $\mathrm{m}$ ) of the corresponding $\sigma_{\theta}$ layer, whereas black boxes delimit the R1-R3 1064 subregions. c) Mean meridional temperature section along $35^{\circ} \mathrm{W}$; black contours depict $\sigma_{\theta}$ levels. d) Mean $\theta / S$ 1065 diagram and main water-masses observed within the 3 white boxes delimited in a). Water-masses: NAW: North 1066 Atlantic Water; SAW: South Atlantic Water; TSW: Tropical Surface Water; EAW: Eastern Atlantic Water; 1067 NACW: North Atlantic Central Water; SACW: South Atlantic Central Water; AAIW: Antarctic Intermediate 1068 Water. 

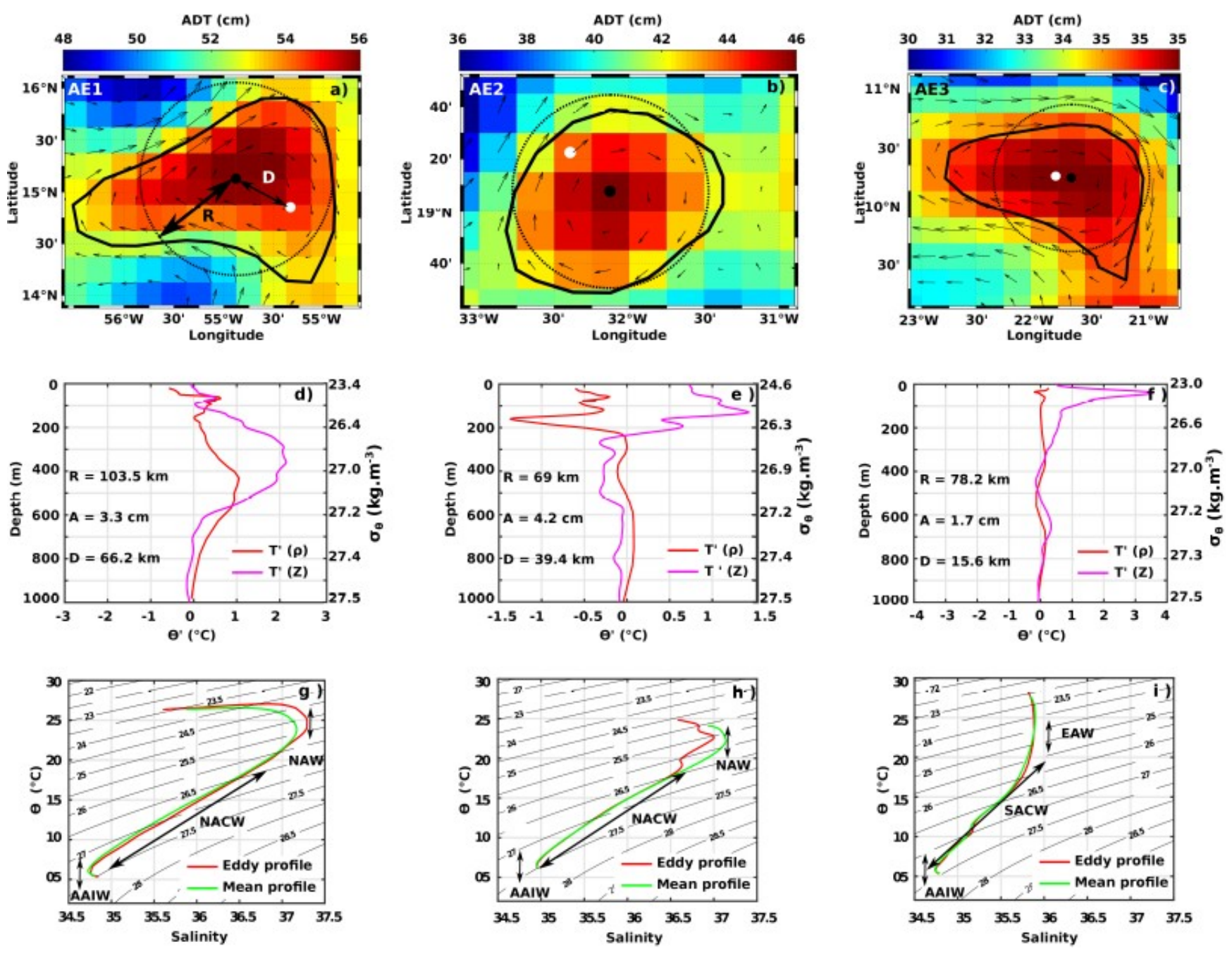

1073 Figure 5. Density-coordinate anomalies compared to depth-coordinate anomalies for three case-study 1074 anticyclonic eddies. a-c) Eddy characteristics in AVISO maps. Black and white dots correspond to the eddy 1075 centers and the location of surfaced Argo floats, respectively, black contours delimit eddy edges whereas dotted 1076 circles represent the contours of the disc corresponding to eddy areas. d-f) Temperature anomalies observed 1077 within eddies in depth-coordinates (magenta lines) and in density-coordinates (red lines). R, A and D indicate 1078 the eddy radius, amplitude and the distance of the surfaced Argo float to the eddy center. g-i) $\theta$-S diagram 1079 obtained within eddies (red lines) and for the mean climatology at the same location (green curves). Water1080 masses: NAW: North Atlantic Water; EAW: Eastern Atlantic Water; NACW: North Atlantic Central Water; 1081 SACW: South Atlantic Central Water; AAIW: Antarctic Intermediate Water. 


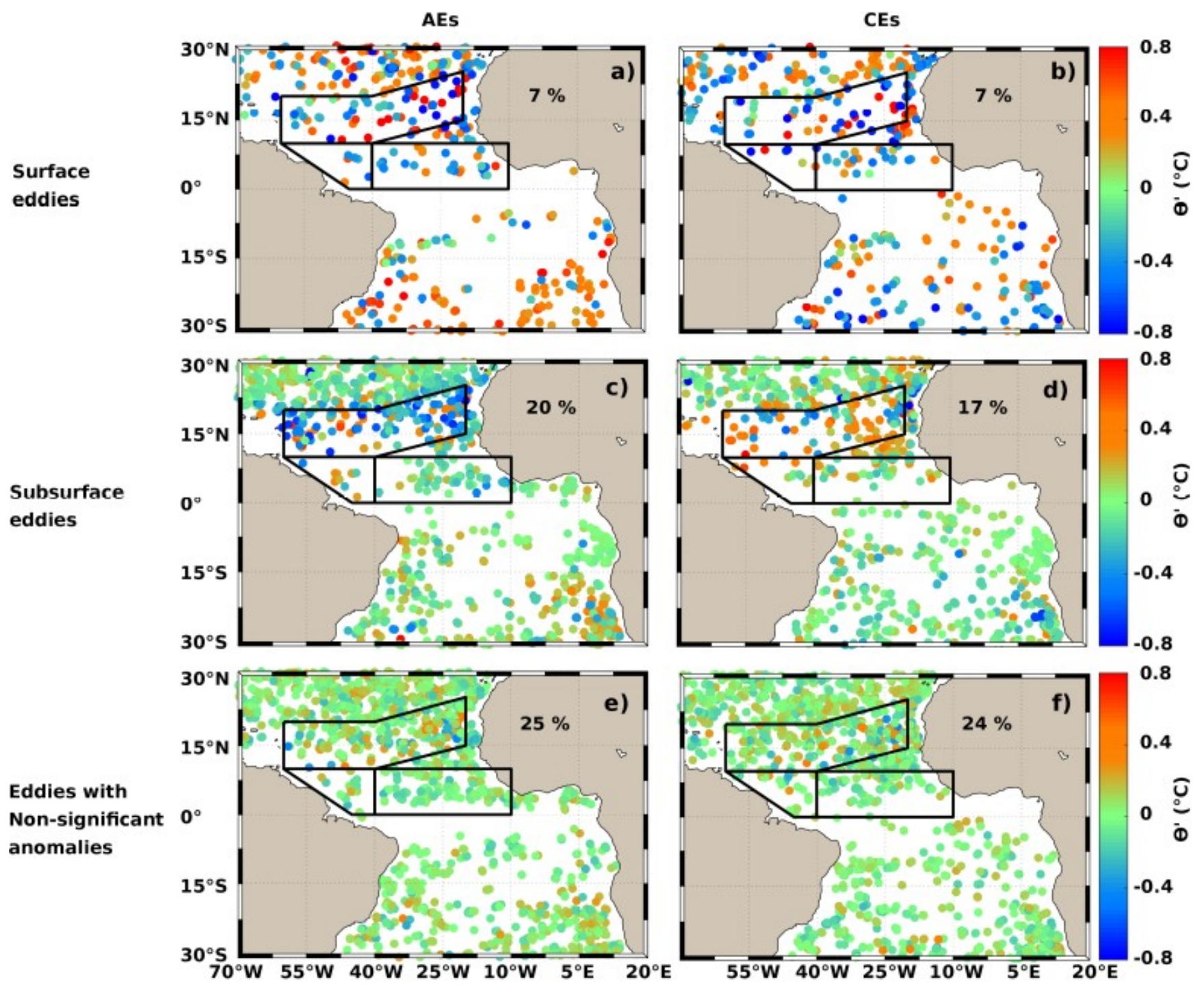

1083 Figure 6. Spatial distribution of isopycnal temperature anomalies within new-born eddies. a-b) Significant 1084 surface $\theta^{\prime}$ within AEs and CEs. c-d) Significant subsurface $\theta^{\prime}$ within AEs and CEs. e-f) non-significant $\theta^{\prime}$ within 1085 AEs and CEs. Black boxes delimit the R1-R3 subregions. Numbers indicate the percentage of sampled eddies 1086 for each category. 
R1
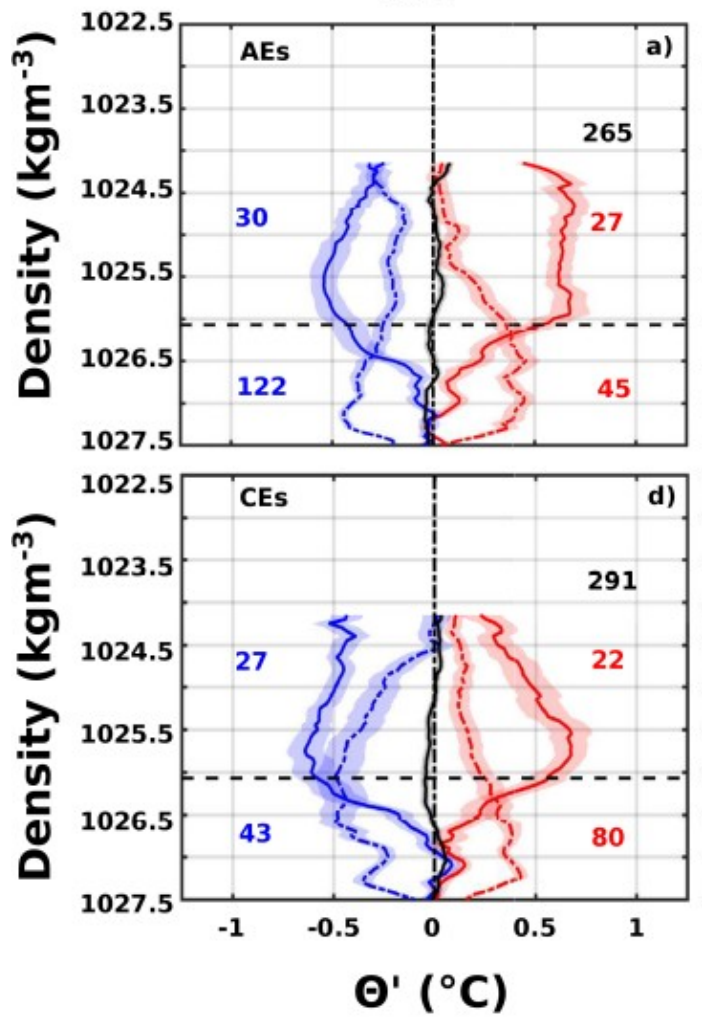

R2
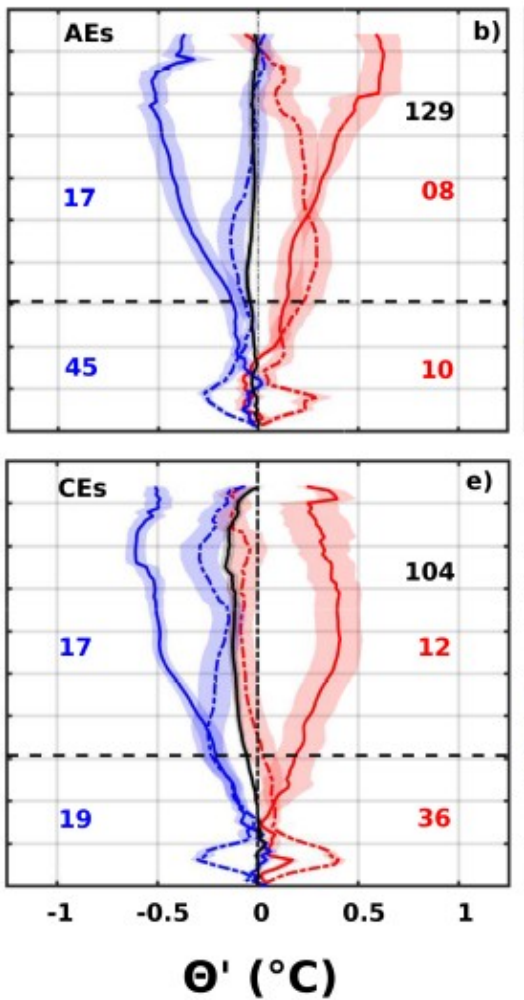

R3
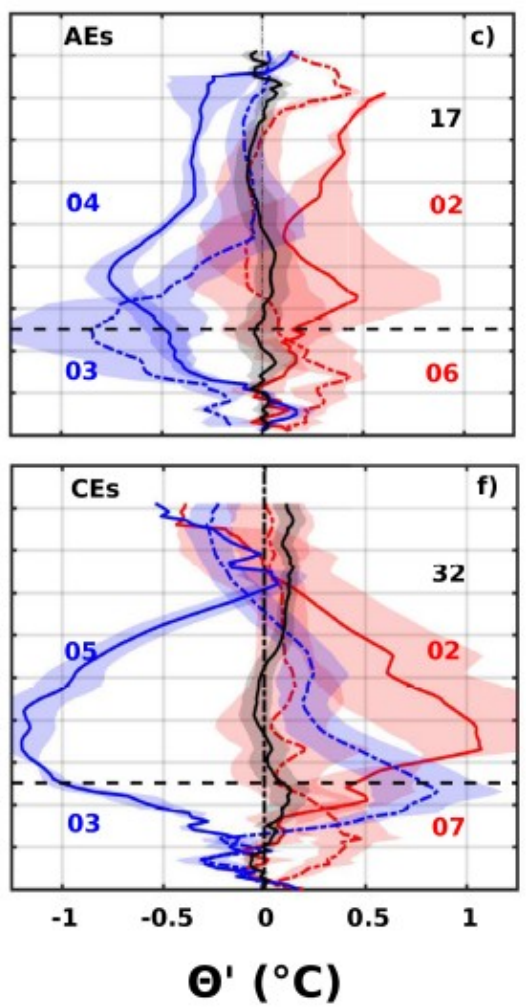

1090

1091

1092

1093

1094

1095

1096

1097

1098

1099

1100

Figure 7. Mean isopycnal temperature structures of newborn a-c) anticyclonic (AEs) and d-f) cyclonic (CEs) eddies within 3 sub-regions (R1-R3.) Significant surface and subsurface anomalies are represented in solid and dashed lines, respectively, whereas positive anomalies are in red and negative anomalies in blue. Non-significant anomalies are represented in black. For each subplot, black numbers indicate the number of sampled eddies having non-significant $\theta^{\prime}$, the right red top (bottom, respectively) numbers indicate the number of surface (subsurface) eddies with positive anomalies and the left blue top (bottom, respectively) numbers indicate the number of surface (subsurface) eddies with negative anomalies. Shading areas correspond to standard errors of the means, evaluated using Student's t test with a significance level of 95\%. For each subregion, the horizontal dashed line corresponds to the base of the pycnocline that delimits the surface and subsurface layers. 
a)

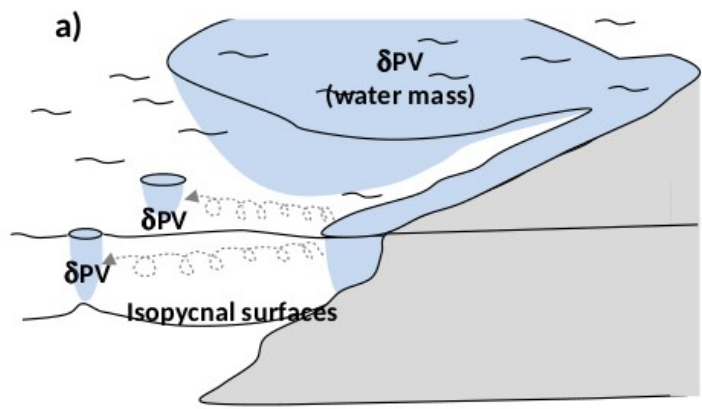

\section{7}

1108

1109

1110

1111

1112

1113

1114

1115

1116

1117

1118

1119

1120 c)
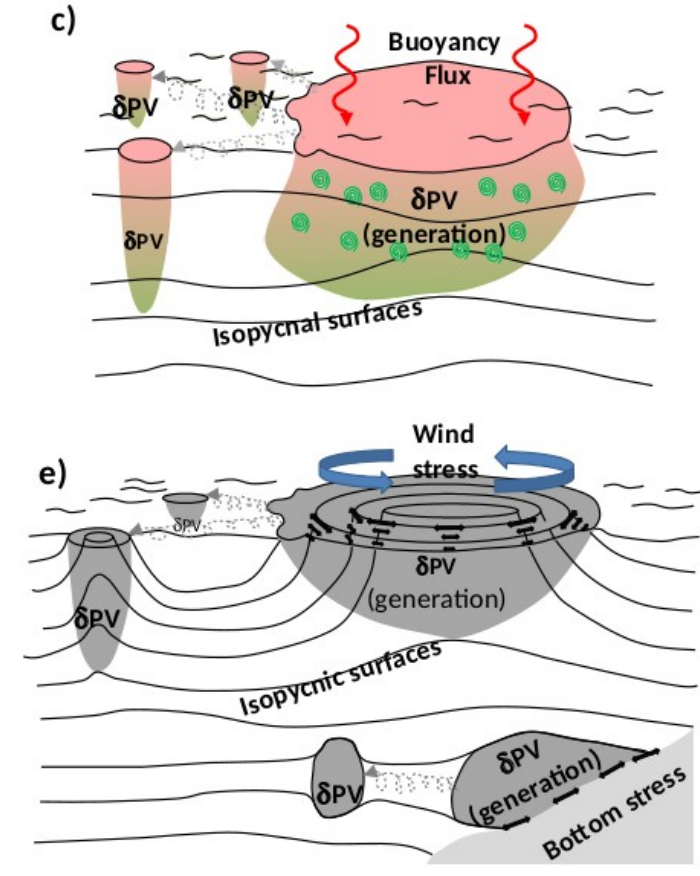

(6) Mixing

W surface buoyancy flux

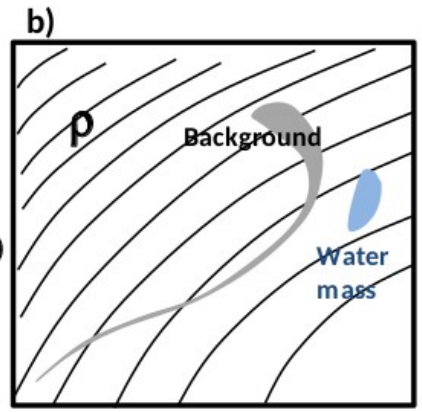

$\mathrm{S}$

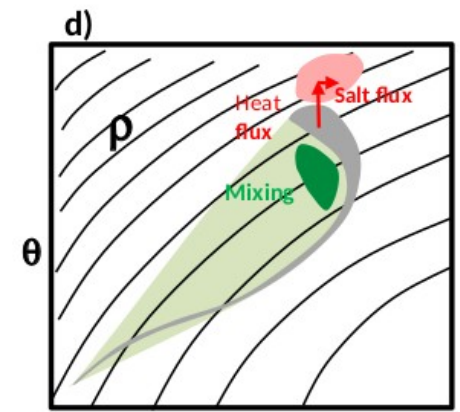

S

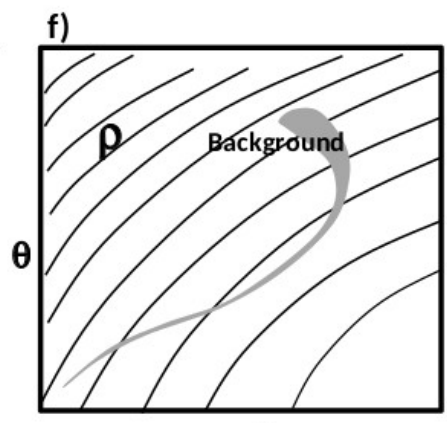

S

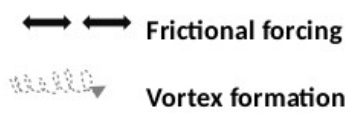

1121 Figure 8. Qualitative analysis of the combined effects of specific mechanisms on the creation of PV (left 1122 column) and corresponding $\theta / S$ diagrams (right column). a-b) Advection of specific water-mass (in blue) in a 1123 region with different PV and $\theta / S$ characteristics (in white/black). c-d) Creation of PV anomalies by diapycnal 1124 mixing (green) and atmospheric buoyancy fluxes (red); These mechanisms also create $\theta / S$ anomalies. e-f) 1125 Frictional effects associated with surface wind or bottom stress; This mechanism is the only one that does not 1126 create $\theta / S$ anomalies. $\theta / S$ diagrams representative of the background are in black. 

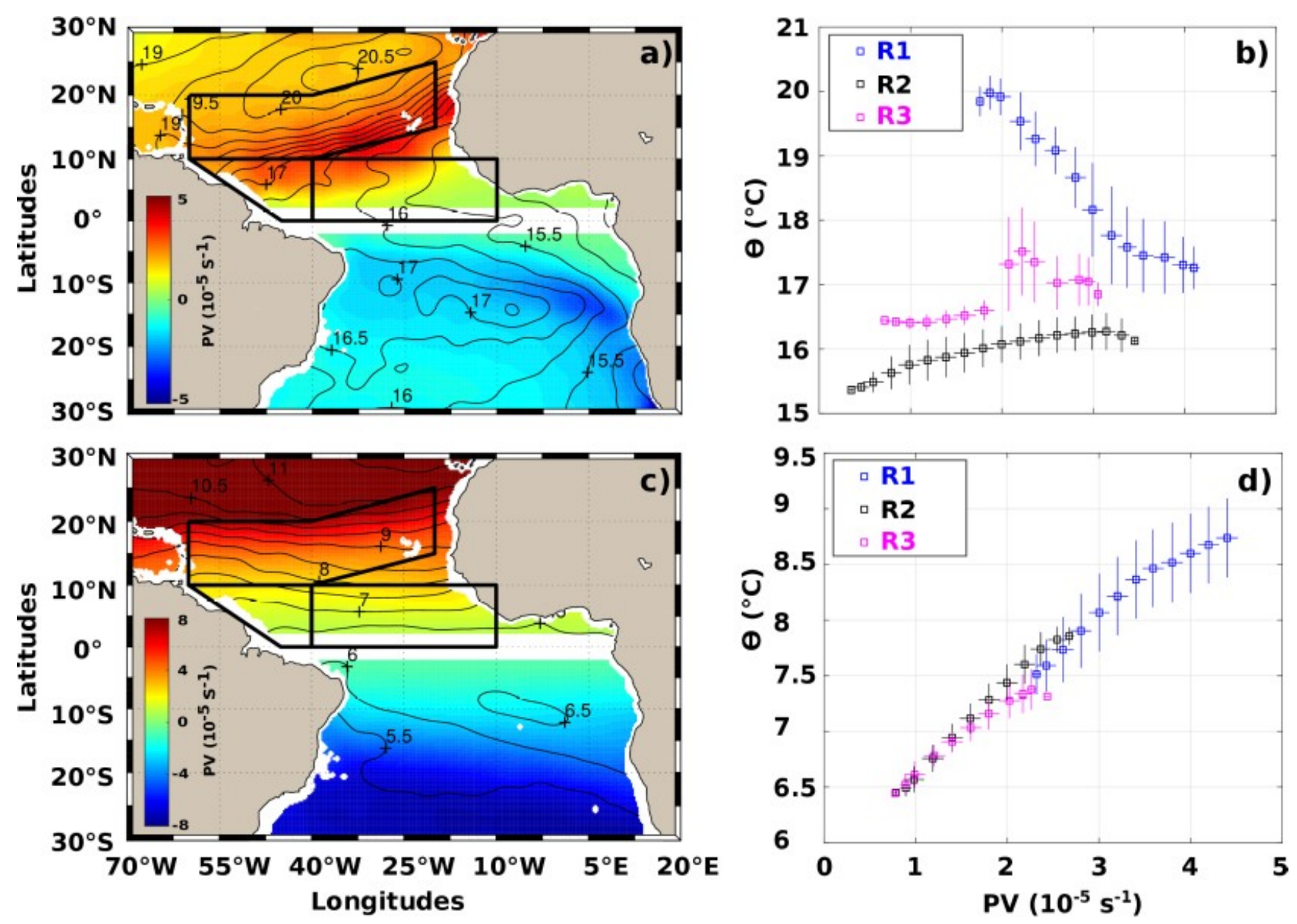

1128 Figure 9. Large-scale rescaled potential vorticity (PV, color shading) and $\theta$ (in ${ }^{\circ} \mathrm{C}$, black contours), averaged 1129 between a) $\sigma_{\theta}=25.75-26.5 \mathrm{~kg} \mathrm{~m}^{-3}$ and c) $\sigma_{\theta}=26.9-27.4 \mathrm{~kg} \mathrm{~m}^{-3}$. Black boxes delimit the R1-R3 subregions. b 1130 \& d) PV/ $\theta$ relationships in R1-R3 subregions. PV (and corresponding $\theta$ ) were averaged in intervals of $0.2 \times 10^{-5} \mathrm{~s}^{-1}$ 1131 (dots) and the corresponding standard deviations are indicated by solid lines. 


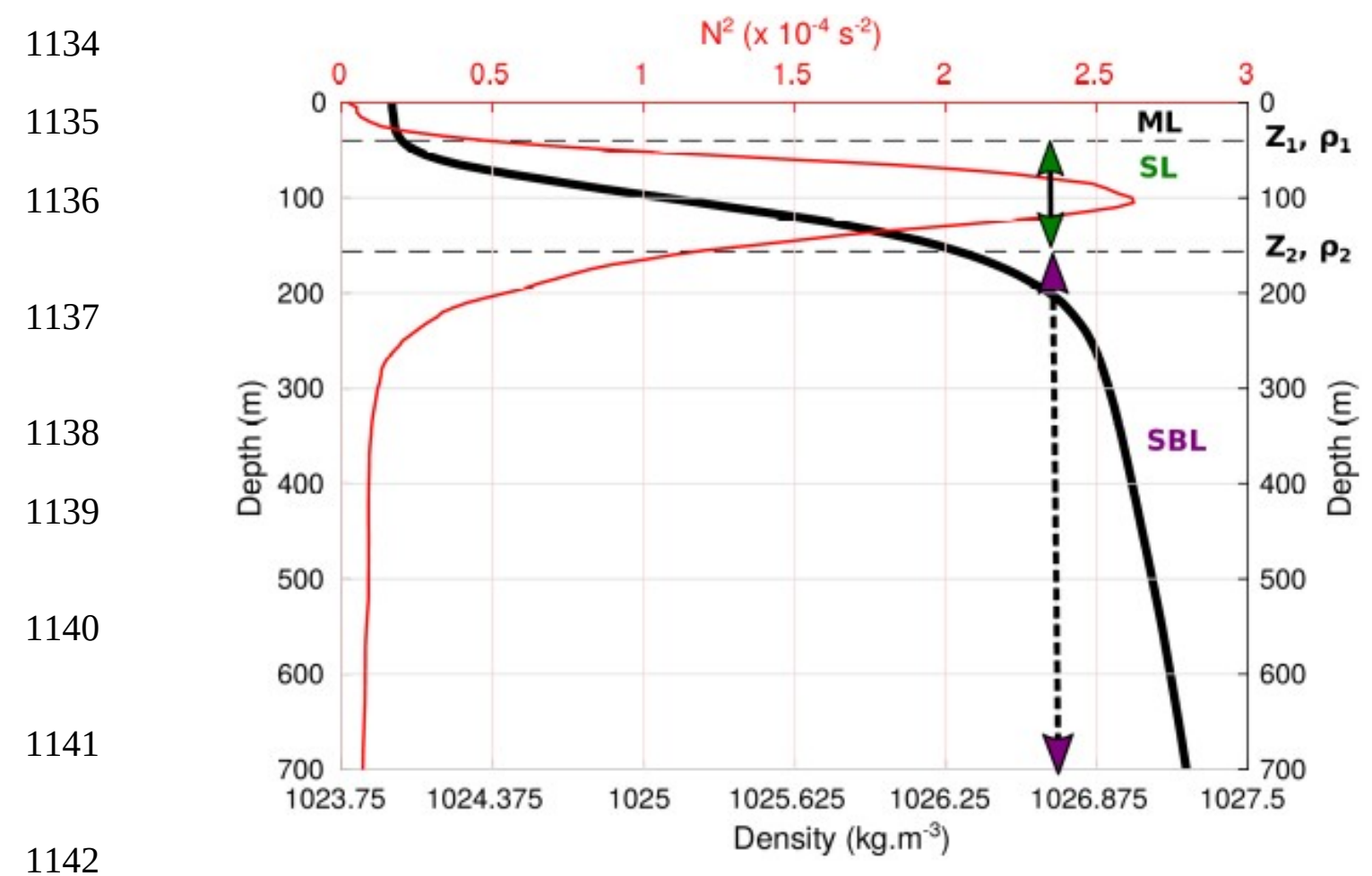

1143 Figure A1. Mean density (black curve) and square buoyancy frequency ( $\mathrm{N}^{2}$, red curve) profiles at a given grid 1144 location showing the mixed (ML), surface (SL) and subsurface (SBL) layers. $\left(\mathrm{Z}_{1}, \rho_{1}\right)$ and $\left(\mathrm{Z}_{2}, \rho_{2}\right)$ are the mixed 1145 layer and the base of the pycnocline depths and densities. 


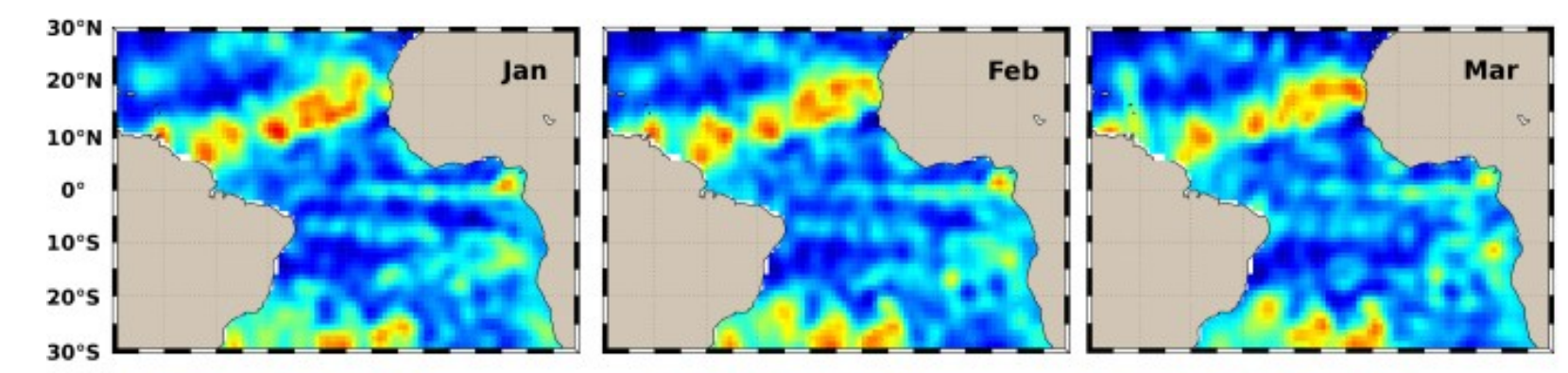




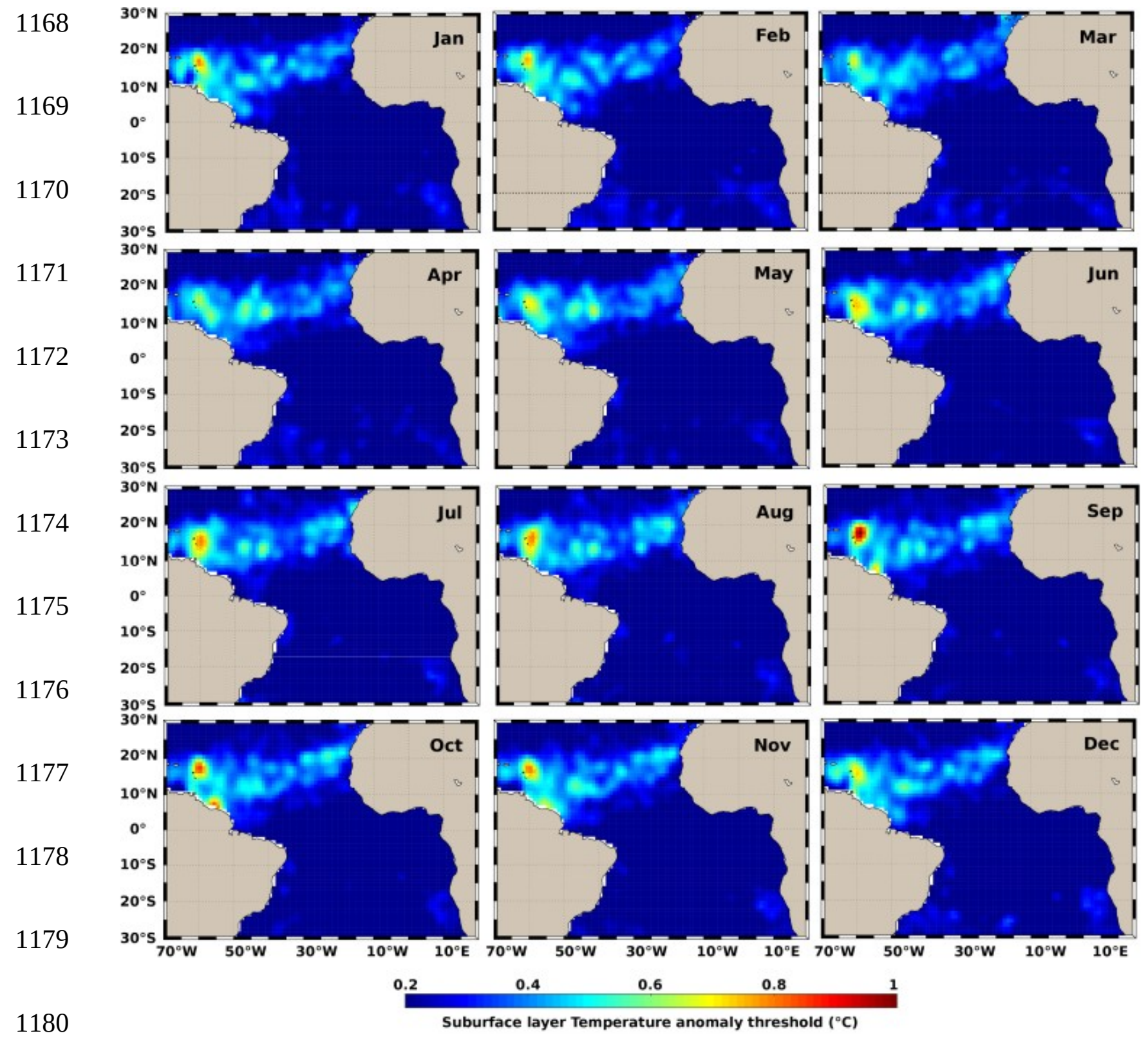

1181

1182 Figure A3. Monthly mean of the temperature anomaly threshold within the subsurface layer.

1183

1184 

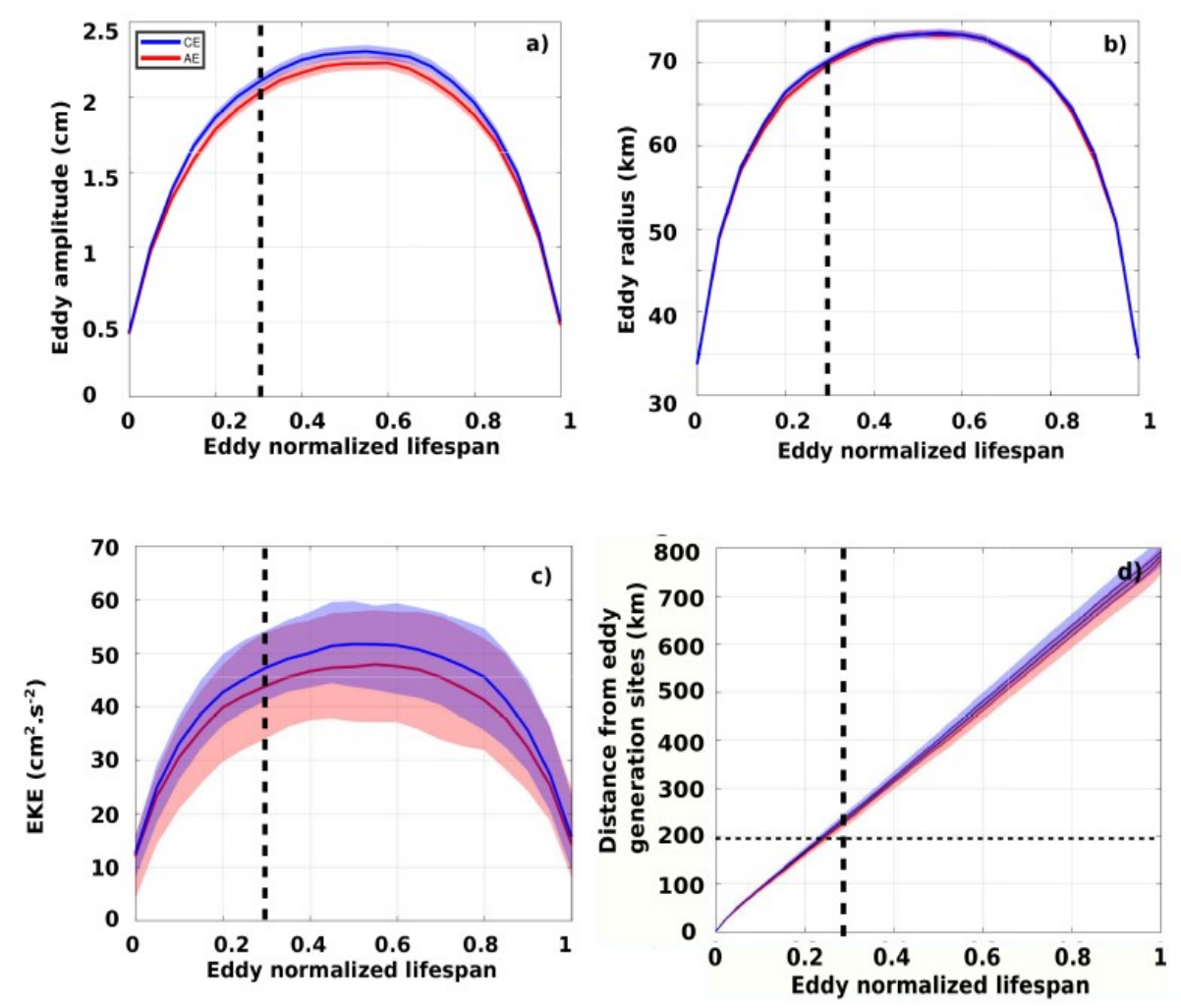

1194 Figure A4. Mean eddy a) amplitude, b) radius, c) kinetic energy (EKE) and d) propagation distance as a function 1195 of normalized lifespan. Vertical dashed lines delimit the eddy growth phase from the mature phase, and 1196 correspond to 30\% of eddy lifespan and the horizontal dashed line indicates the $200 \mathrm{~km}$ distance. Shading areas 1197 correspond to standard errors around the mean. 
Figure1. 
Figure2. 
Figure3. 


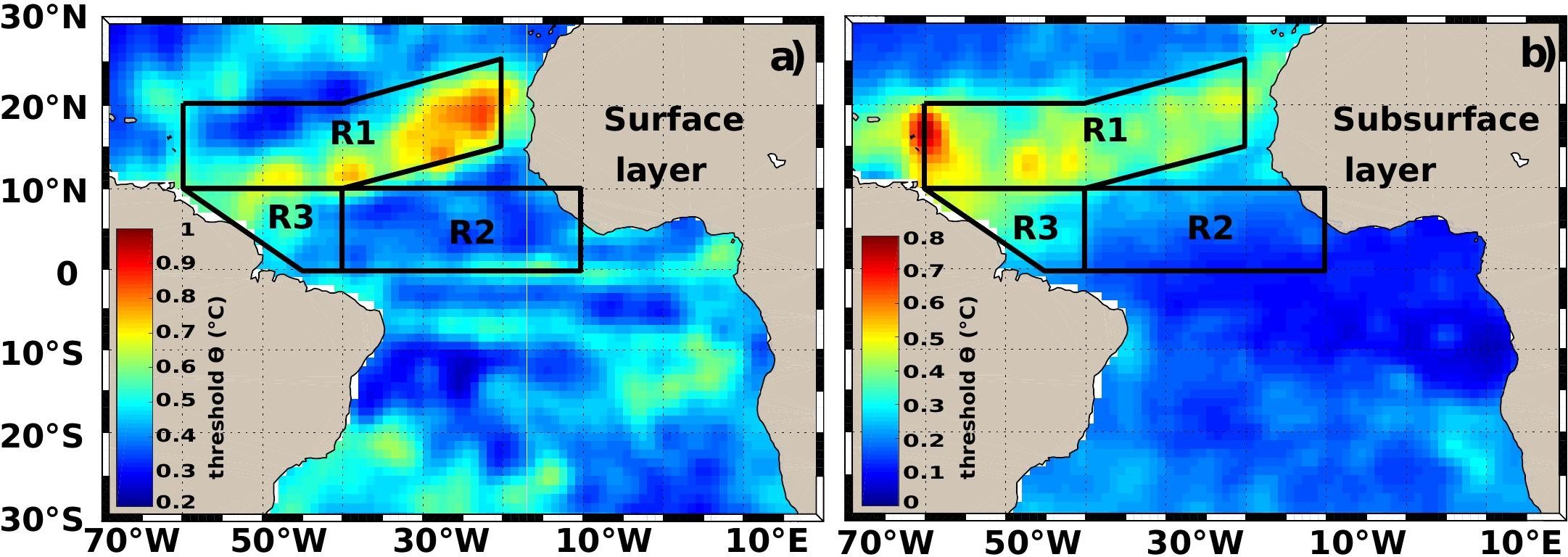


Figure4. 
Figure5. 


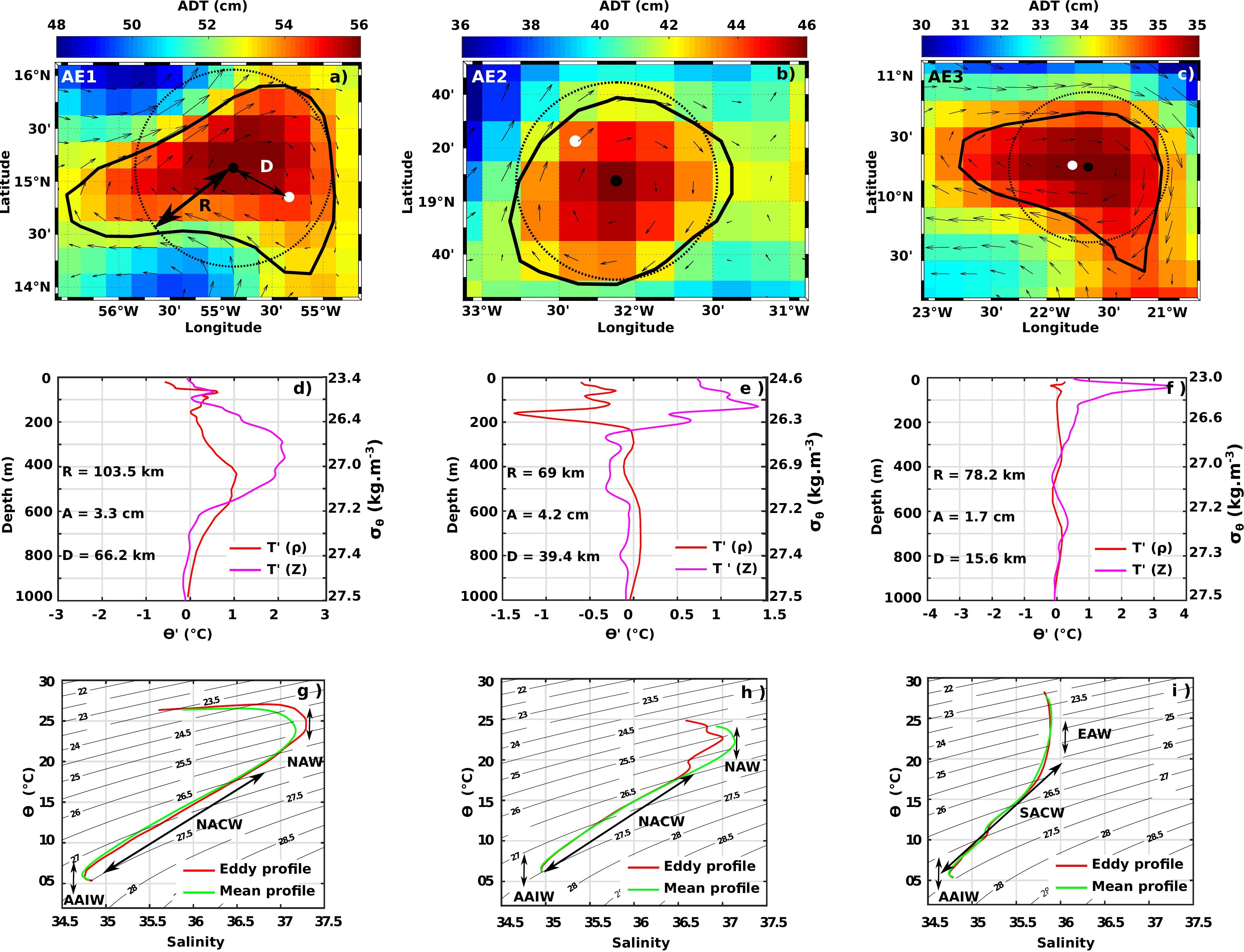


Figure6. 
Surface eddies

Subsurface eddies

Eddies with Non-significant $0^{\circ}$ anomalies

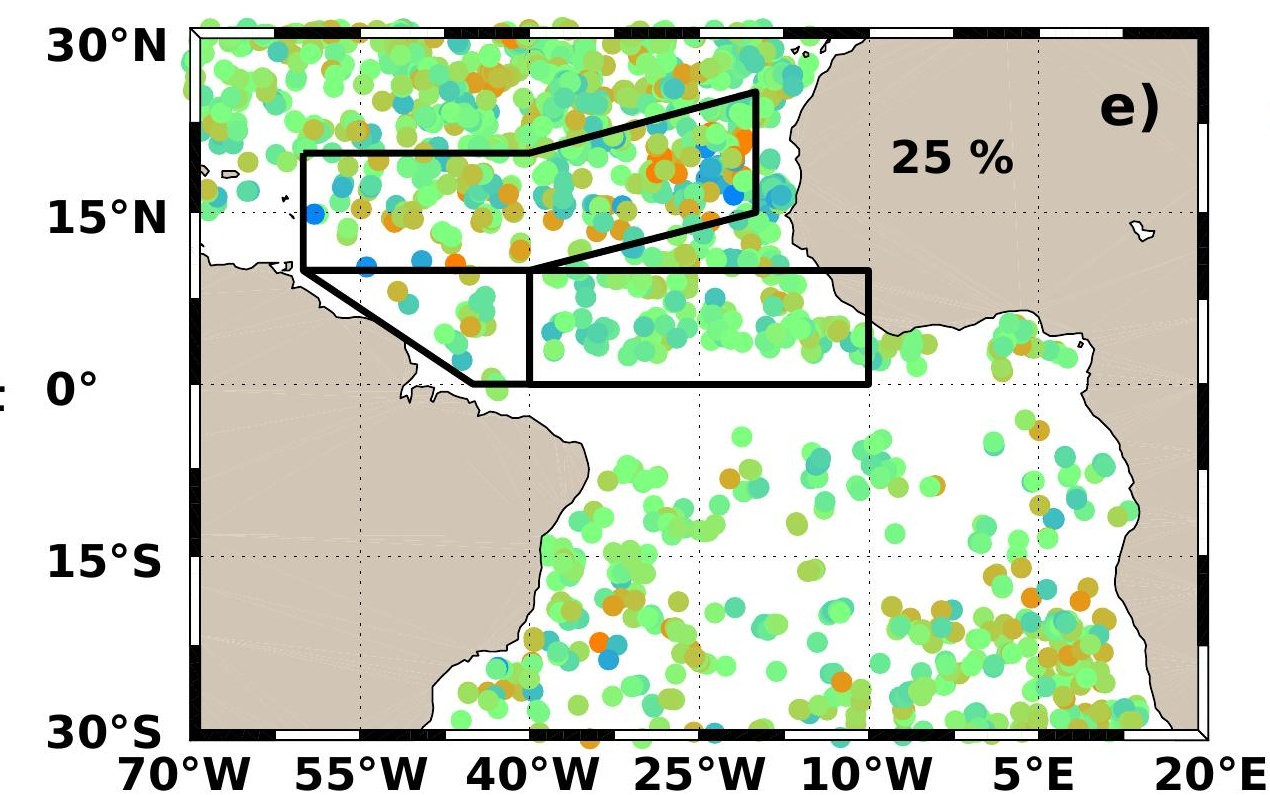

c)

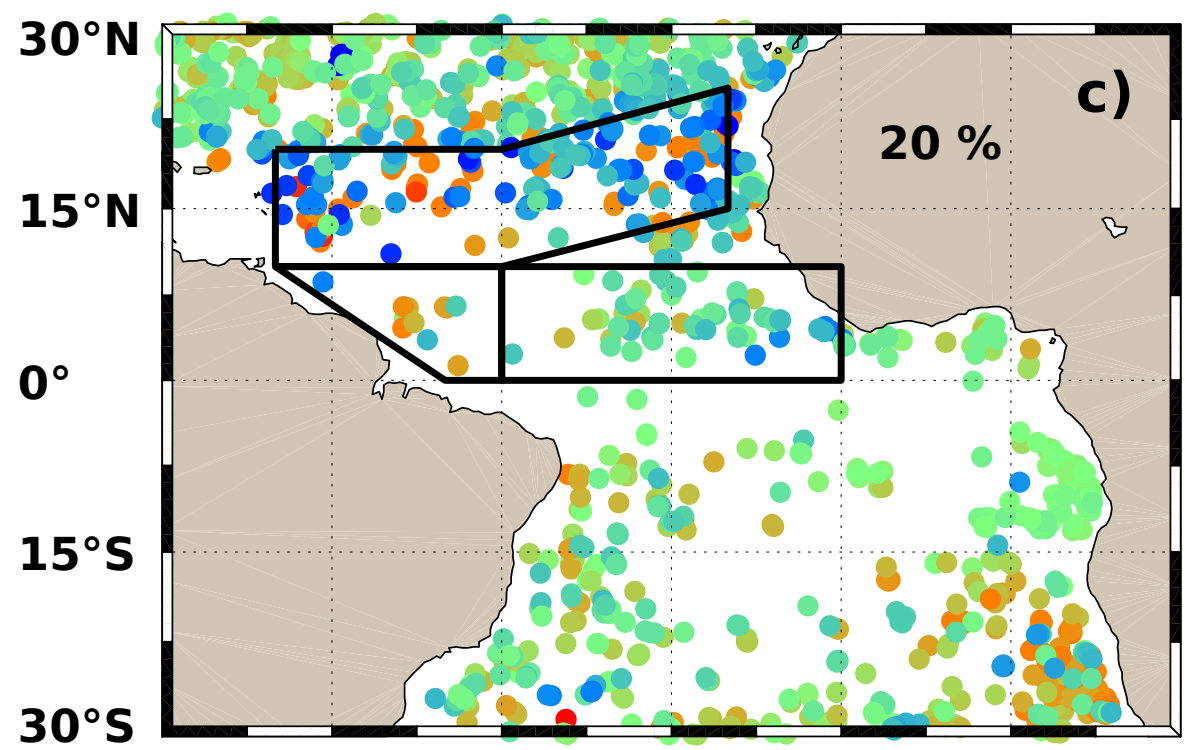

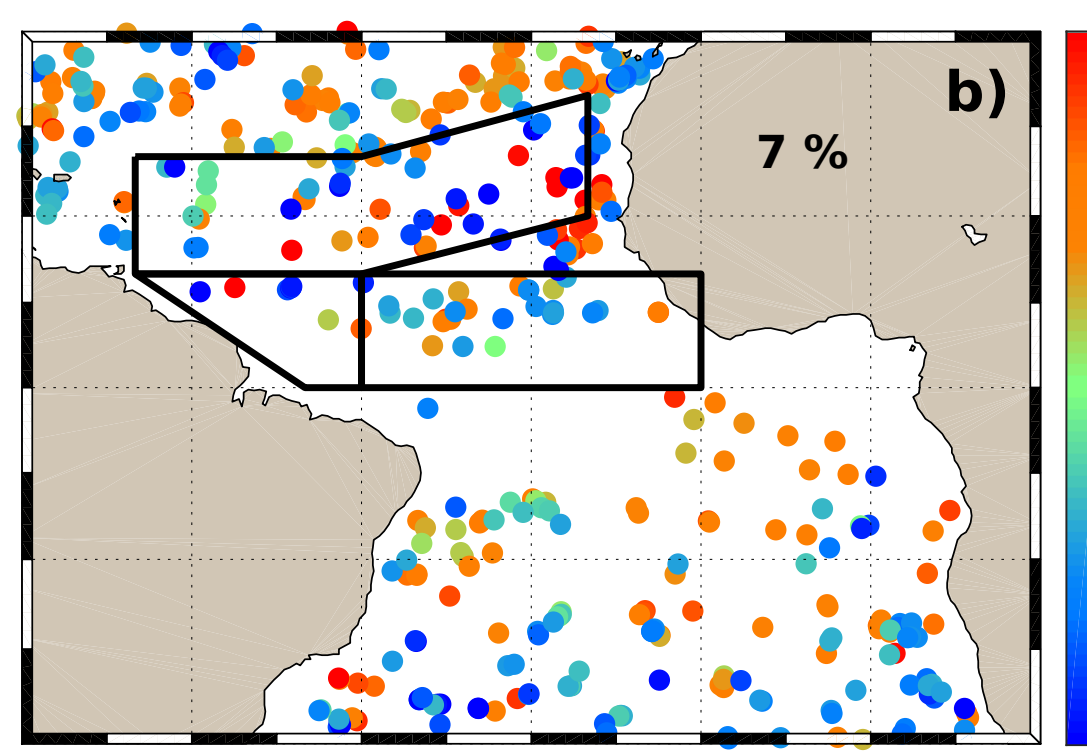

0.8
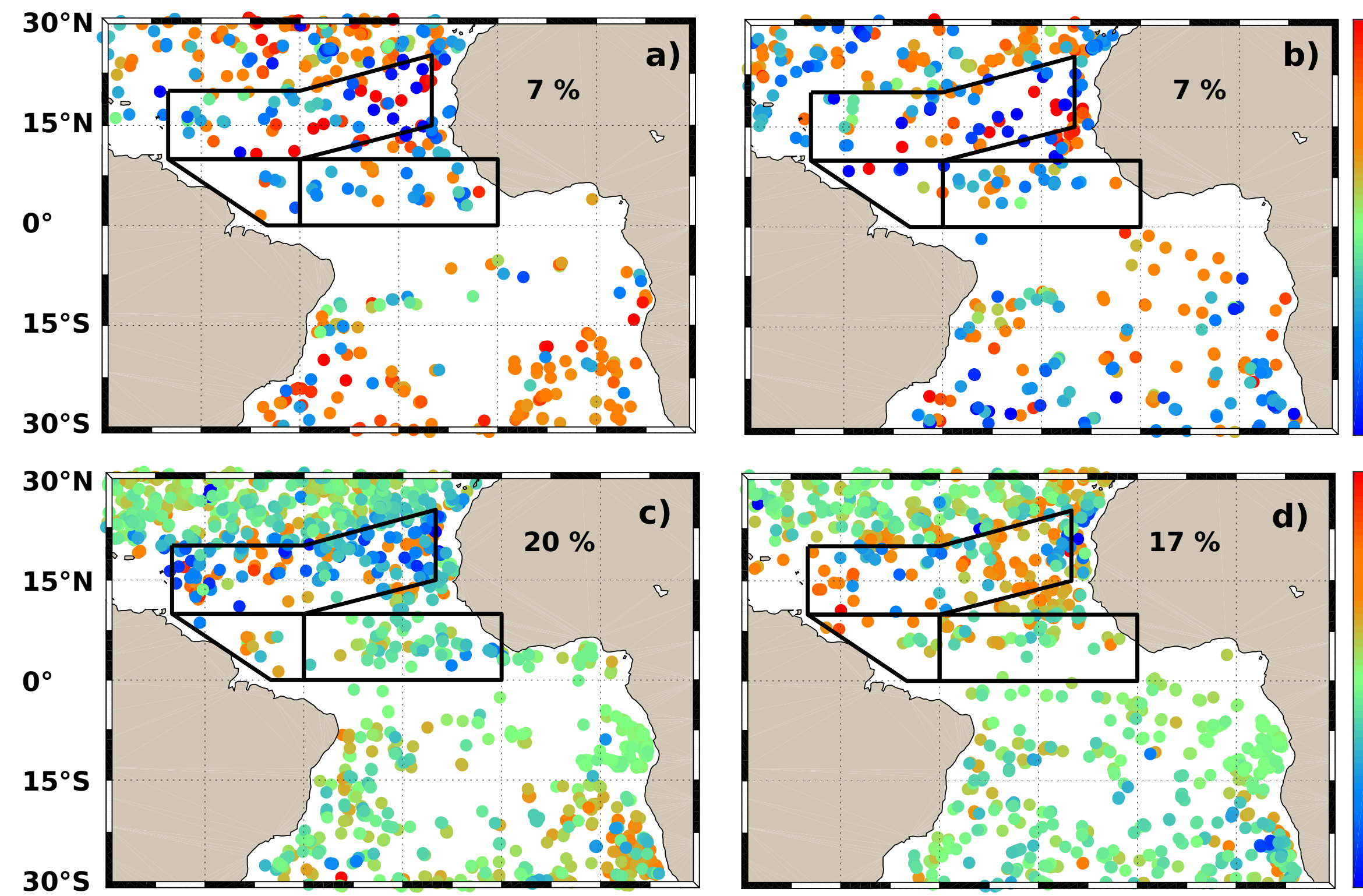

$-0.8$

0.8

0.4

0
0.
0
0
0

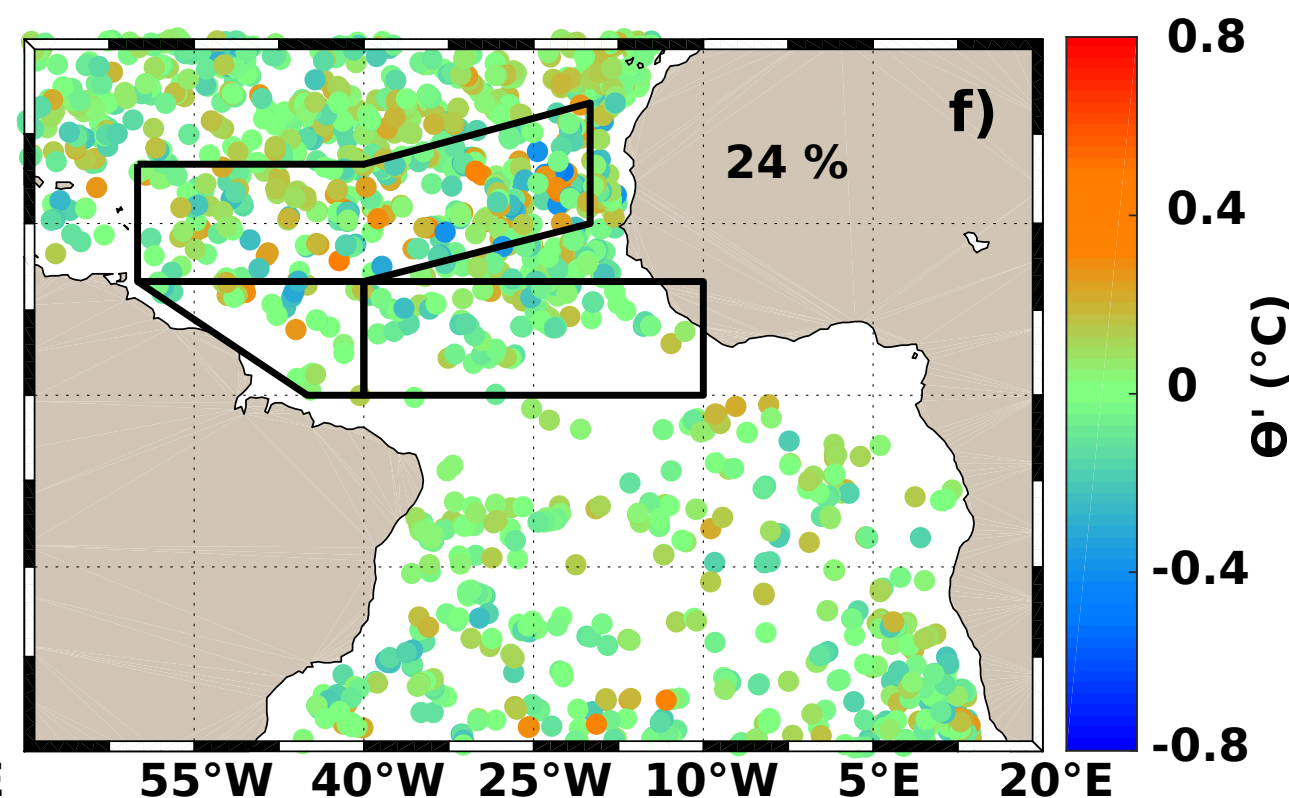


Figure7. 
Figure8. 

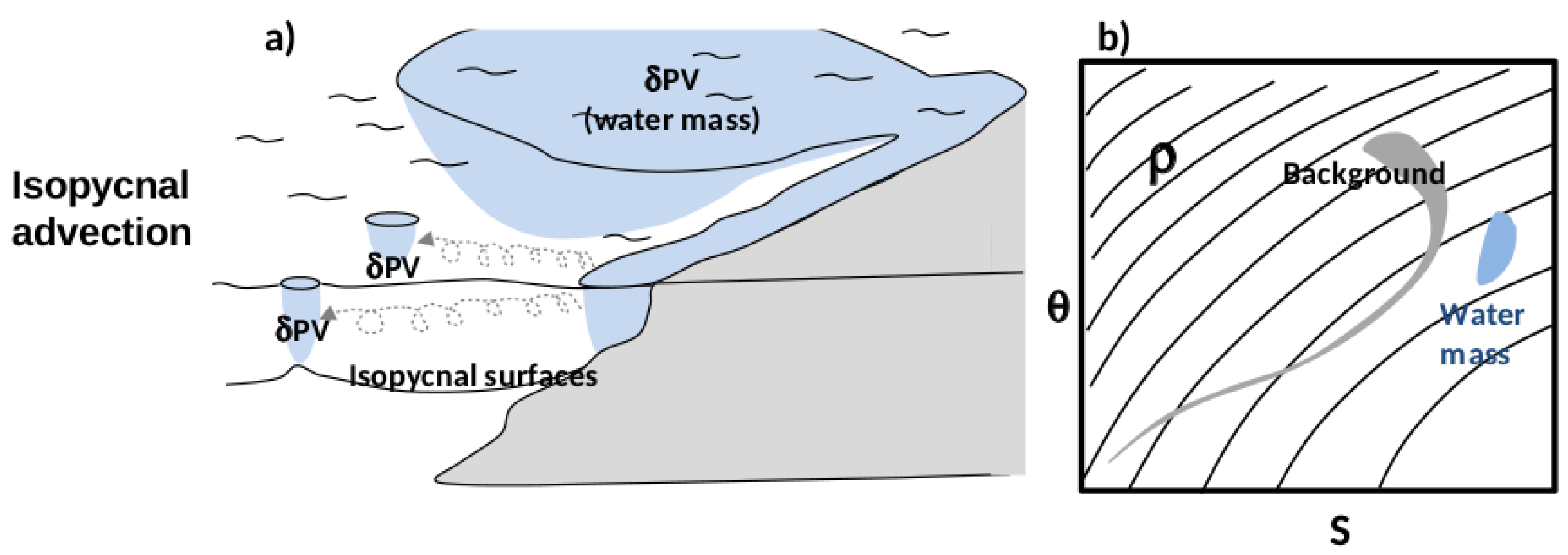

\section{Mixing effects}

c)

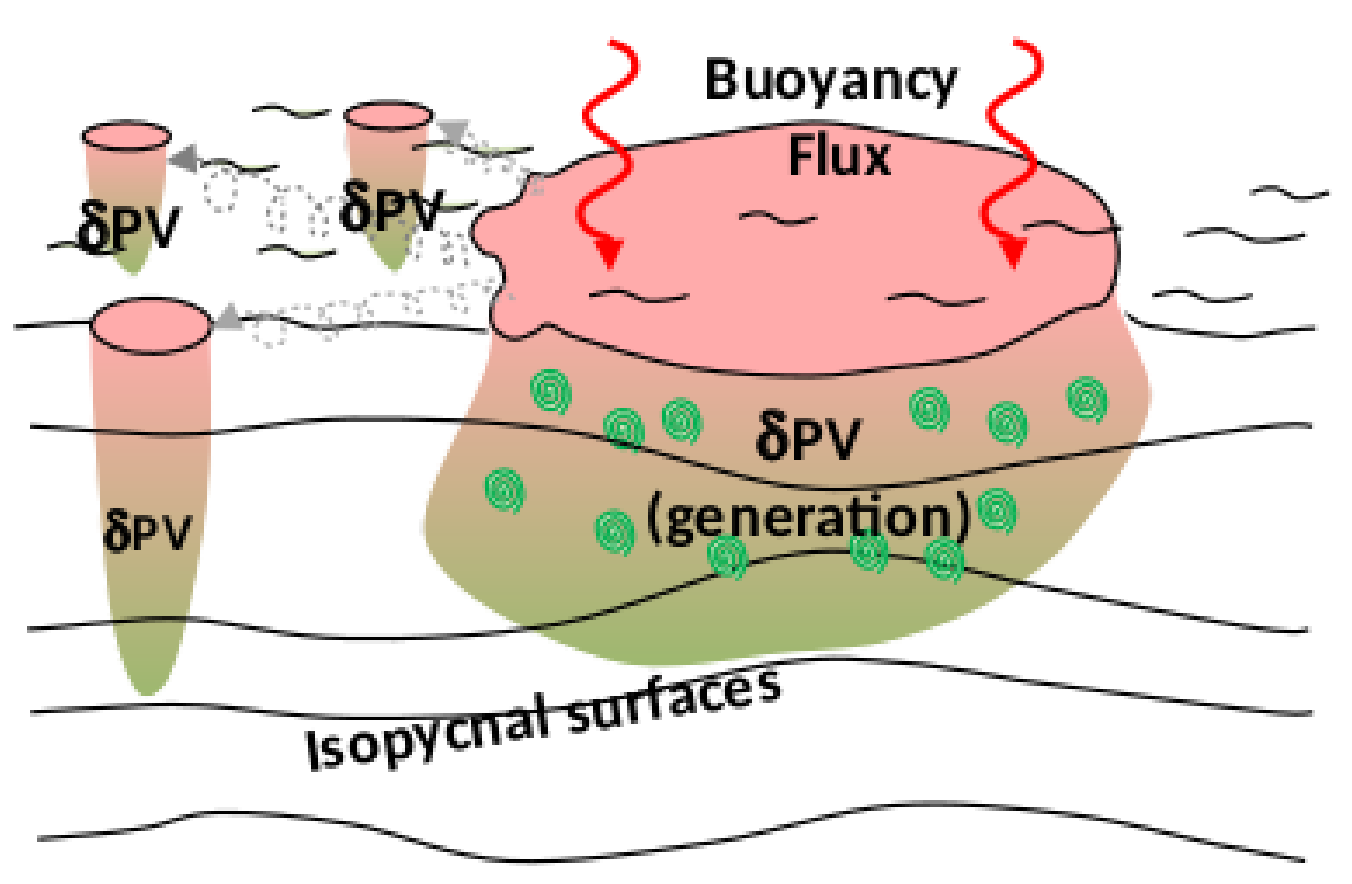

\section{Frictional effects}
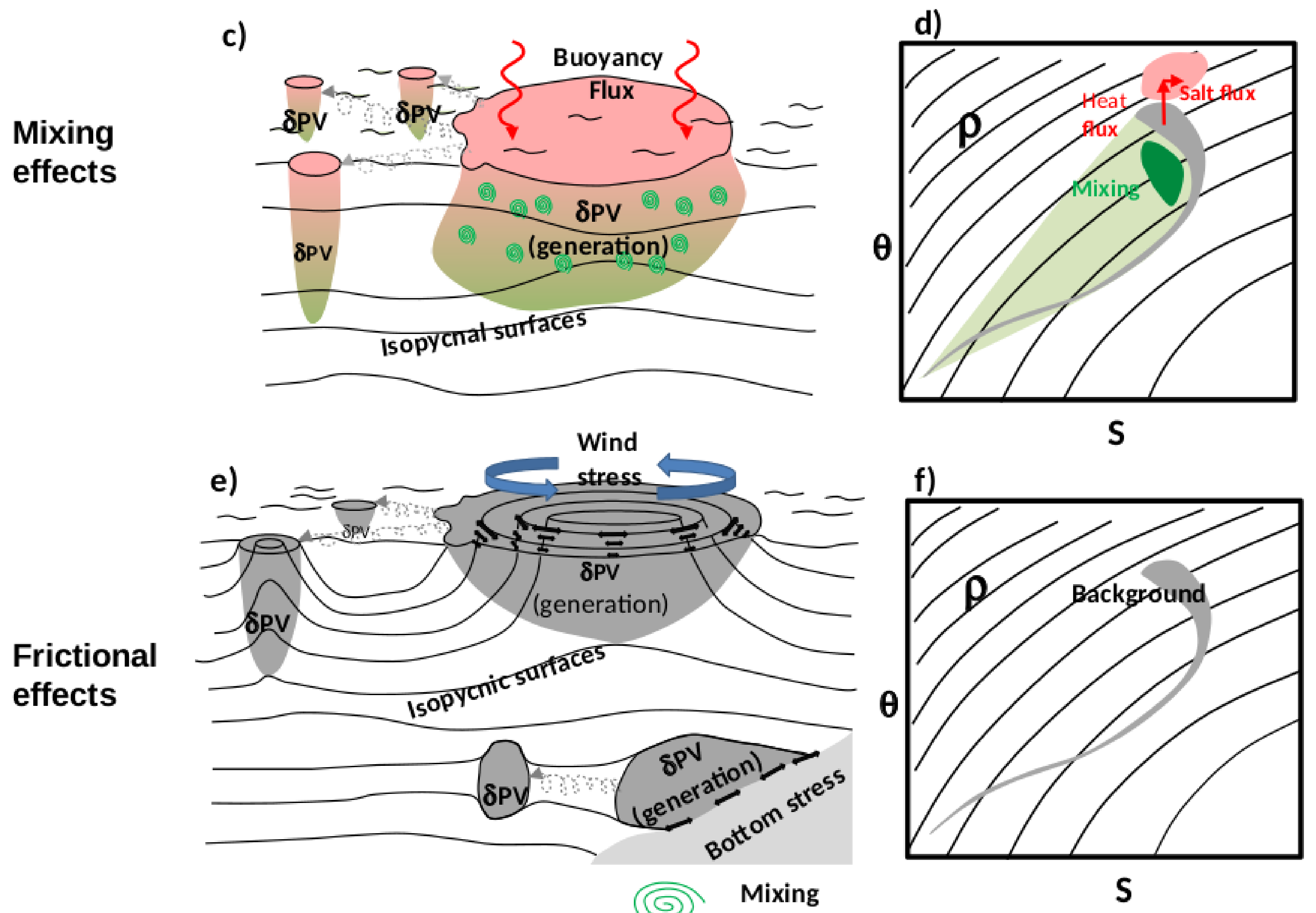

W surface buoyancy flux

$\longrightarrow \longrightarrow$ Frictional forcing

Wh - Vortex formation 
Figure9. 
Figure A1. 
$\mathrm{N}^{2}\left(\times 10^{-4} \mathrm{~s}^{-2}\right)$

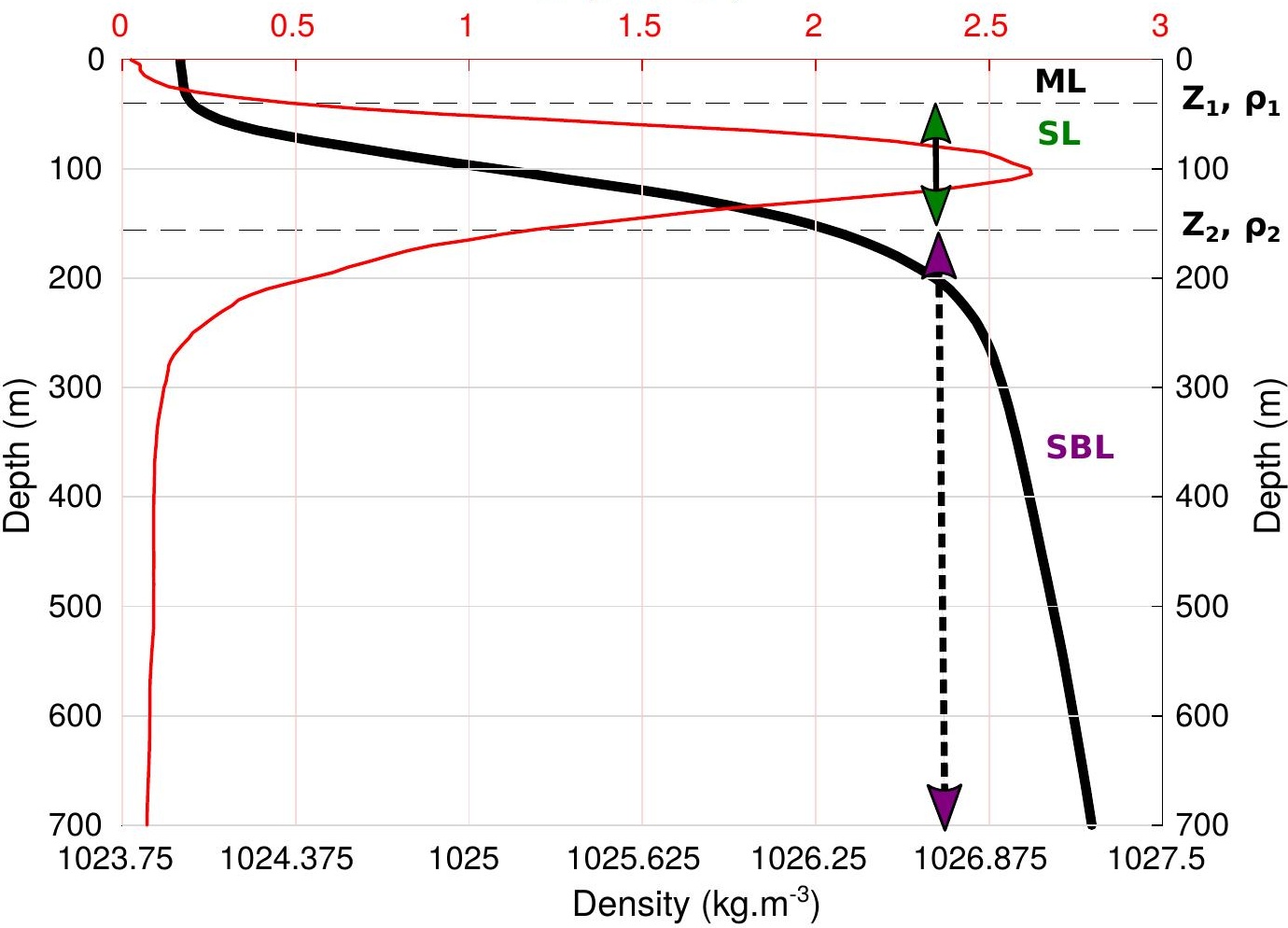


Figure A2. 


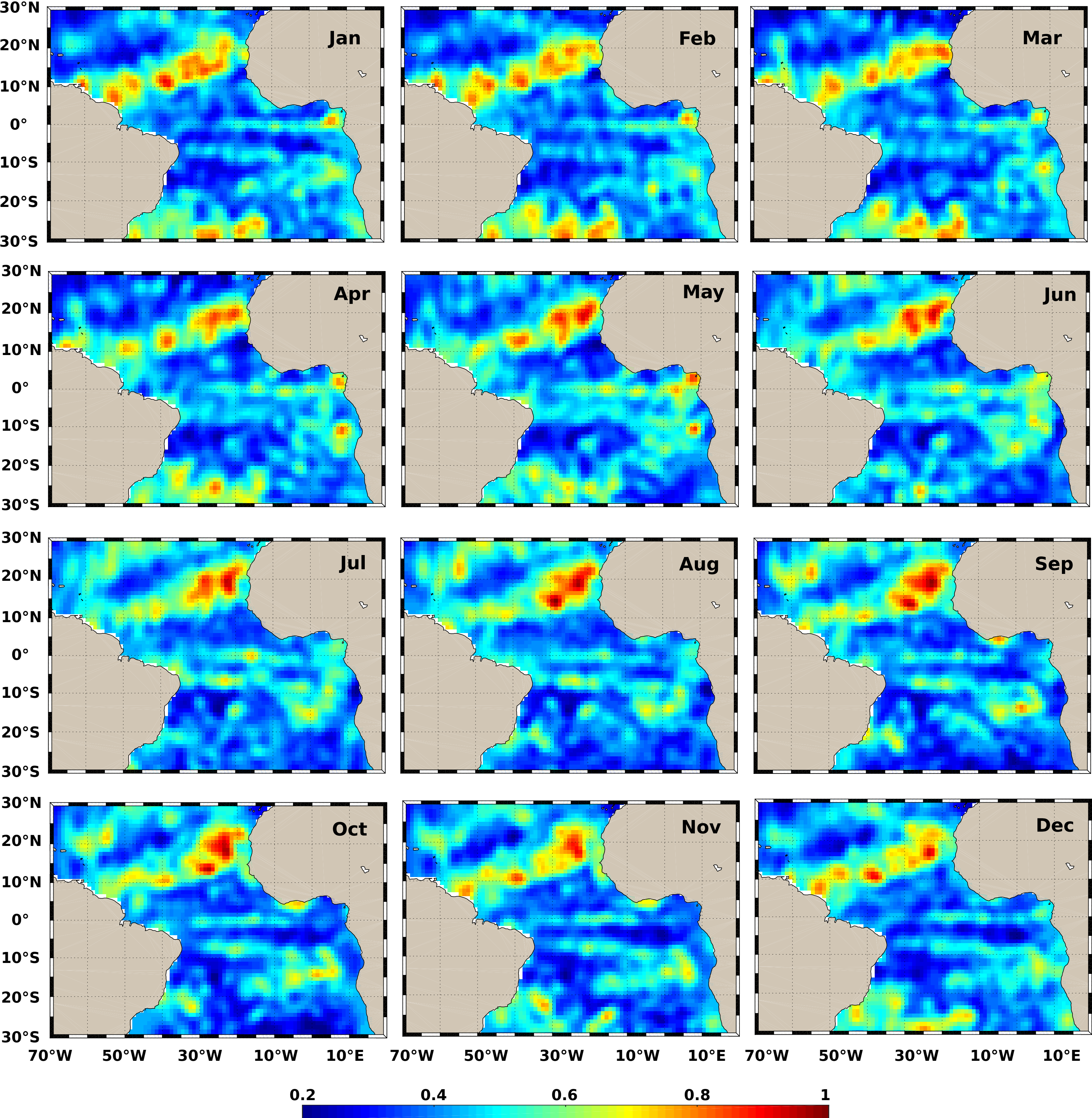

Surface layer Temperature anomaly threshold $\left({ }^{\circ} \mathrm{C}\right)$ 
Figure A3. 


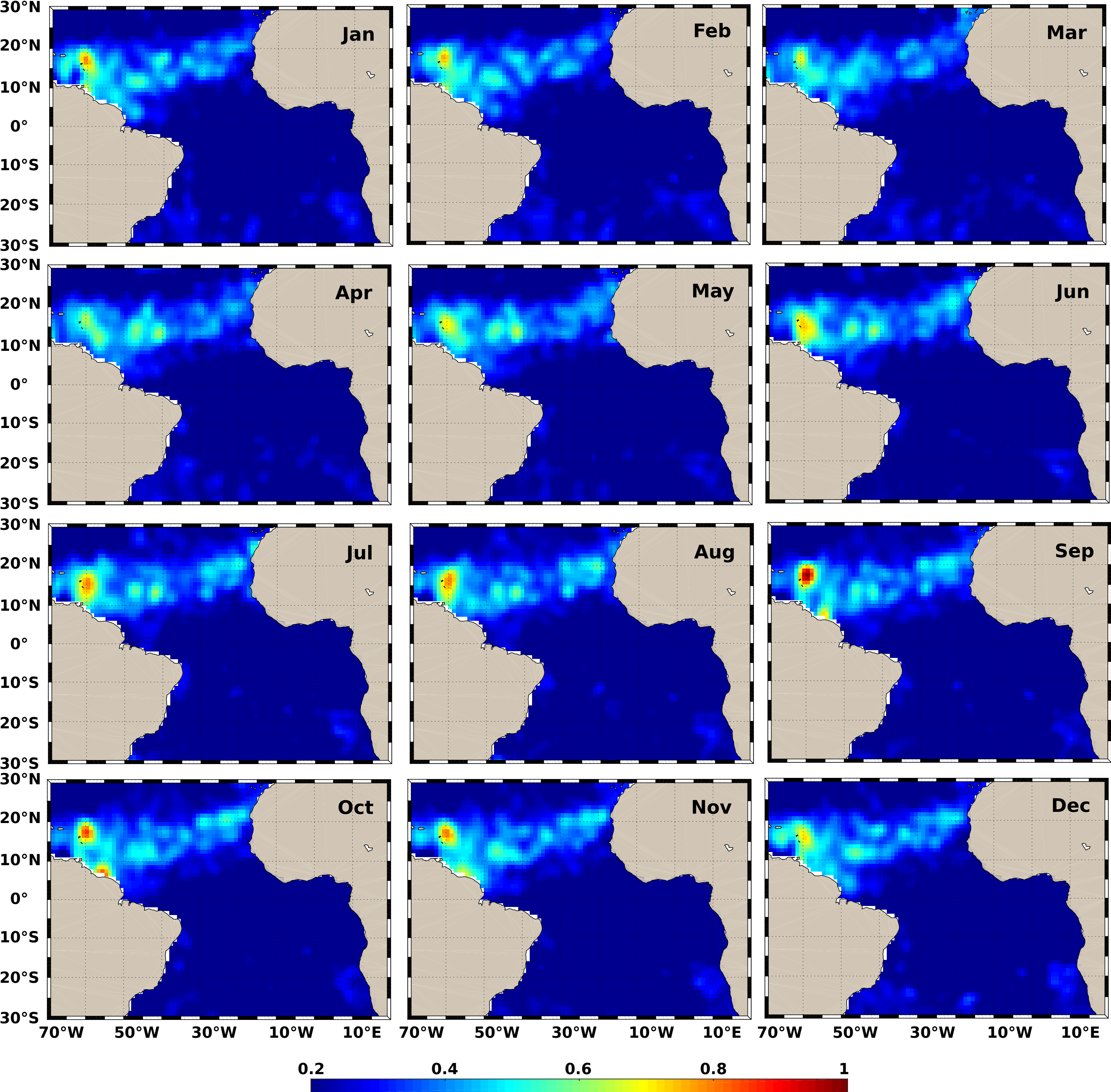

Suburface layer Temperature anomaly threshold $\left({ }^{\circ} \mathrm{C}\right)$ 
Figure A4. 

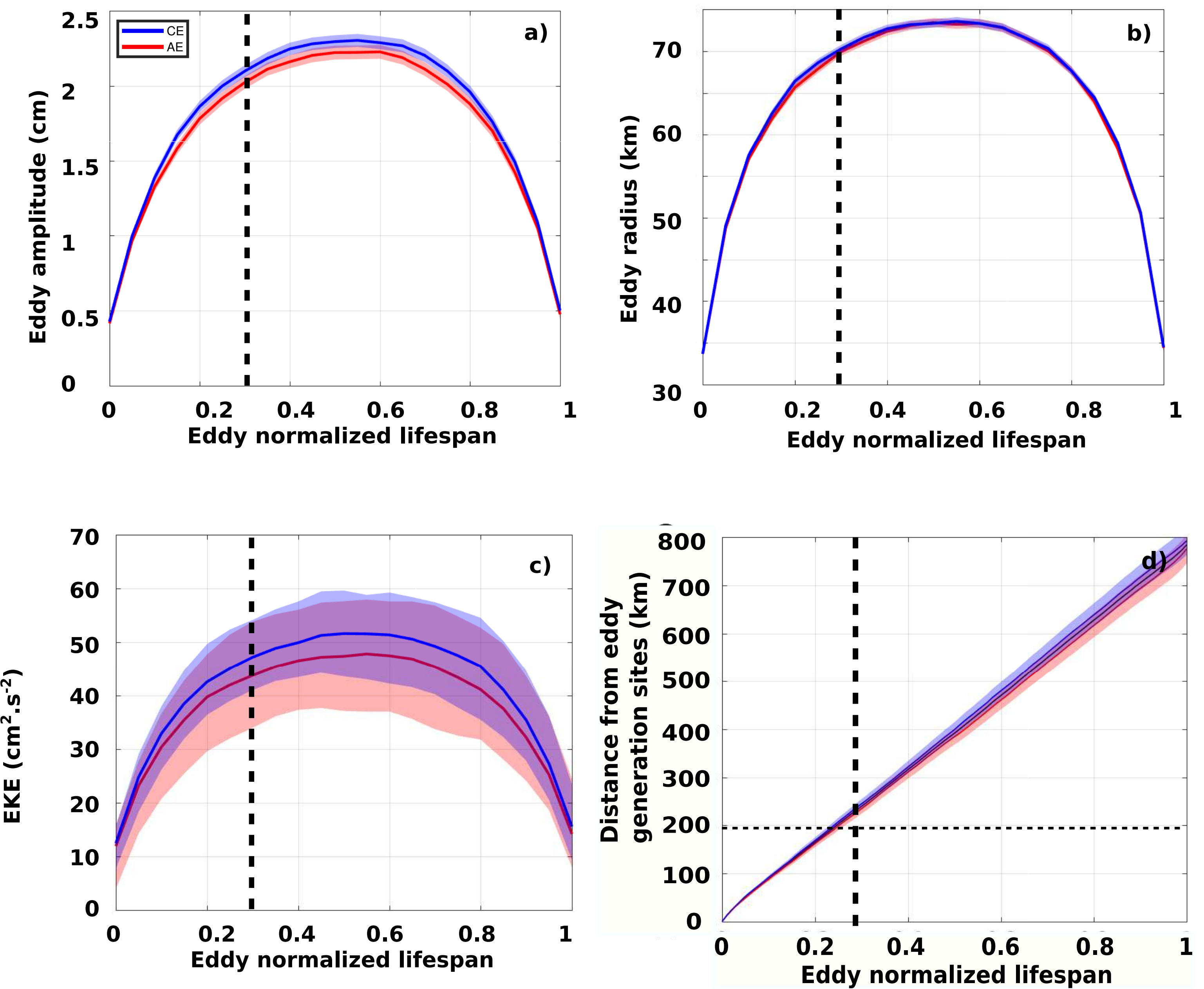